\title{
EVALUATION OF CUMULATIVE RANDOM SHOCKS GENERATED FROM A SEMI-MARKOV MODULATED POISSON PROCESS AND ITS APPLICATION TO CDO PRICING
}

\author{
Jia-Ping Huang \\ VU University Amsterdam
}

\author{
Ushio Sumita \\ University of Tsukuba
}

(Received July 16, 2011; Revised February 16, 2012)

\begin{abstract}
During the past decade, a structured financial product called "Collateralized Debt Obligation (CDO)" has been drawing much attention of researchers and practitioners, and is now traded with growing liquidity. However, the approach for CDO pricing has been rather limited in the literature, largely because it is necessary to evaluate the time dependent distribution of the underlying cumulative loss so as to find the pricing scheme satisfying the non-arbitrage condition of the derivatives market. The purpose of this paper is to fill this gap by describing the CDO model in terms of a semi-Markov modulated Poisson process. Based on the theoretical results of Huang and Sumita [6] as well as the Laguerre transform method, numerical algorithms are developed for evaluating the time dependent distribution of the cumulative loss up to time $t$, which in turn enables one to evaluate the price of a CDO tranche. Some numerical results are presented, demonstrating the power of the algorithms.
\end{abstract}

Keywords: Applied probability, semi-Markov modulated Poisson process, reward process, Laguerre transform, finance, CDO pricing

\section{Introduction}

A structured financial product called "Collateralized Debt Obligation (CDO)" securitizes a reference portfolio of default risky instruments. If the portfolio contains loans or bonds, then the CDO is called a cash CDO. When the referenced portfolio consists of "Credit Default Swaps (CDS's)", it is called a synthetic CDO. Recently, portfolio credit derivatives such as Tranched Index have been drawing much attention of researchers and practitioners, and are now traded with growing liquidity.

A typical approach for assessing (synthetic) CDO and Tranched Index is based on the Gaussian copula model of $\mathrm{Li}[10]$. Because this model is essentially static, the dynamic evolution of CDO tranches cannot be captured. In order to overcome this difficulty, it is often necessary to introduce a two layer process, where the first layer describing the macro economic condition would affect portfolio loss distributions expressed in the second layer. In other words, the default intensity function would satisfy a stochastic differential equation (or assumed to be driven by a certain Markov chain) characterized by the state of the external economic condition as well as the history of defaults up to the current time. Some recent papers along this direction include Arnsdorf and Halperin [1], Bielecki, Vidozzi and Vidozzi [2], Frey and Backhaus [5], de Kock, Kraft and Steffensen [3], Schönbucher [11] and Sidenius, Piterbarg and Andersen [12].

Although some structural characteristics of CDO tranches could be captured by these pioneering models, they are far from offering the computational vehicle for evaluating CDO tranches in continuous time, and one often has to resort to Monte Carlo simulation for this 
purpose. To the best knowledge of the authors, Lando [9] is the first to suggest the use of MMPP for capturing the stochastic behavior of portfolio losses. (Here, MMPP stands for Markov Modulated Poisson Process, which is a generalization of the Poisson process in that the arrival rate depends on the state of an independent continuous time Markov chain. For further details, the reader is referred to Fischer and Meier-Hellstern [4].) Consequently, the computational procedure for general Markov chains can be applied for evaluating loss distributions. However, the infinitesimal generator characterizing the underlying Markov chain could be huge, imposing the tremendous computational burden. Takada, Sumita and Takahashi [21] recapture the MMPP model of Lando [9] within the context of CDO. By exploiting the first passage time structure, efficient computational procedures are developed for evaluating loss distributions and pricing CDO tranches. This model has been extended further in Takada and Sumita [20], where two industrial segments are introduced to enhance the reality of the model. In both Takada, Sumita and Takahashi [21] and Takada and Sumita [20], the loss of a single default is considered to be constant, which may be somewhat unrealistic.

In order to overcome this pitfall, this paper provides a cumulative random shock process generated from a semi-Markov modulated Poisson process, enabling one to cope with random costs associated with corporate defaults. It should be noted that this process is a special case of the multivariate reward process discussed in Huang and Sumita [6], where the transform results obtained therein are used to derive the time dependent distribution of the cumulative aggregate loss explicitly. Using the Laguerre transform method developed by Keilson and Nunn [7], Keilson, Nunn and Sumita [8], and further studied by Sumita [13], computational algorithms are developed for evaluating the distribution of the loss process for the underlying CDO model, which in turn enables one to evaluate the price of a CDO tranche.

The analytic results obtained in Huang and Sumita [6] cannot be inverted into real domain. Accordingly, the use of such results have been largely restricted to asymptotic expansions of moments. However, for applications like the CDO pricing problem under consideration here, the moment information is not sufficient and it is absolutely necessary to evaluate the distribution of the cumulative aggregate loss at time $t$ explicitly. The thrust of this paper is to re-express the transform results by taking advantage of a case of a semi-Markov modulated Poisson process in such a way that the inversion from the bivariate Laplace transform domain to the real domain becomes possible. This work is quite laborious and challenging, and should be considered as a good contribution in its own right. Furthermore, it is worth noting that the bivariate version of the matrix Laguerre transform is developed for the first time in this paper.

Throughout the paper, vectors and matrices are underlined and double underlined respectively, e.g. $\underline{u}$ and $\underline{\underline{v}}$. The vector of having all components equal to 1 is denoted by $\underline{1}$. The indicator function $\delta_{\{\text {Statement\} }}$ takes the value of 1 if Statement holds true and 0 otherwise.

The structure of this paper is as follows. In Section 2, the structure and pricing scheme of CDO are introduced. Section 3 describes the model employed in this paper to capture the default phenomenon, with transform results of the distribution of the cumulative aggregate loss $Z(t)$ of the underlying reference portfolio. Numerical algorithms for evaluating the distribution of $Z(t)$ via the Laguerre transform are provided in Section 4. Some numerical results are presented in Section 5, demonstrating the power of the algorithms, with concluding remarks given in Section 6.

In order to facilitate the readability of the paper, the inversion procedures via the Laguerre transform and the algorithms discussed in Section 4 are summarized in Appendices $\mathrm{A}$ and $\mathrm{B}$. The reader is also referred to Sumita and Kijima $[17,18]$ for a succinct summary 
of the computational algorithms associated with the Laguerre transform.

\section{Structure of CDO and Pricing CDO Tranches}

The CDO scheme involves the protection buyer (the CDO issuer) and the protection seller (the investor) as depicted in Figure 1. Given a reference portfolio, the CDO is typically structured by dividing the associated credit risk into tranches of increasing seniority. Here, each tranche consists of an attachment point $K_{a}$ and a detachment point $K_{d}$ of the cumulative aggregate loss of the underlying reference portfolio, where $0<K_{a}<K_{d}$. The protection buyer is fully responsible for the portfolio loss up to $K_{a}$. When the portfolio loss exceeds $K_{a}$, the protection seller compensates the protection buyer by paying the exceeding amount beyond $K_{a}$ but only up to $K_{d}-K_{a}$. In exchange, predetermined premiums are paid to the protection seller by the protection buyer according to a prespecified schedule up to the maturity year in such a way that no-arbitrage condition of the credit derivatives market is satisfied. Following Takada, Sumita and Takahashi [21], the procedural details are described below for determining the premium scheme for each tranche.
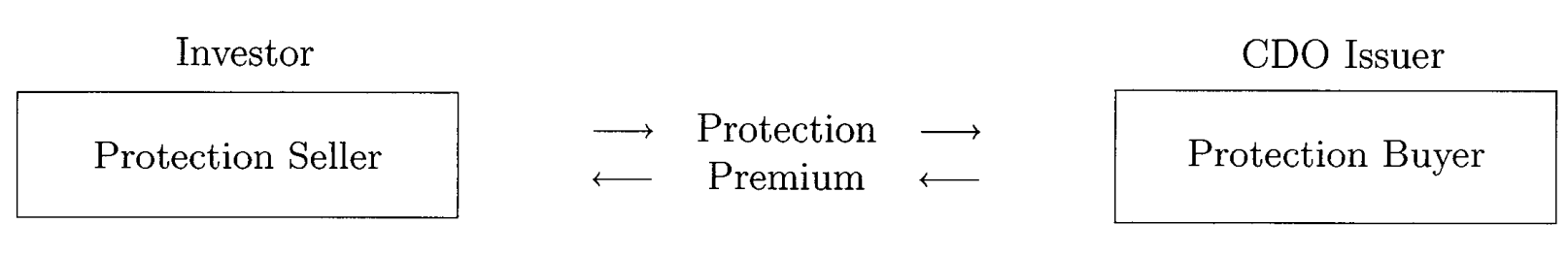

Figure 1: Contract of CDO tranche

A typical CDO tranche contract consists of the attachment point $K_{a}$, the detachment point $K_{d}$, the premium settlement points $\underline{\tau}=\left\{\tau_{0}, \cdots, \tau_{K}\right\}$ with $\tau_{0}=0$, and the unit premium $c_{\left[K_{a}, K_{d}\right]}$. These entities are related to each other through the following procedural details. Let $Z(t)$ be the cumulative aggregate loss of the underlying reference portfolio up to time $t$. We note that $Z(t)$ can be typically described as a stochastic process characterized by a counting process associated with corporate defaults and random bad debts resulting from such corporate defaults. Let $L_{\left[K_{a}, K_{d}\right]}(t)$ be defined by

$$
L_{\left[K_{a}, K_{d}\right]}(t)= \begin{cases}0 & \text { if } Z(t) \leq K_{a} \\ \ell(t)-K_{a} & \text { if } K_{a}<Z(t) \leq K_{d} \\ K_{d}-K_{a} & \text { if } K_{d}<Z(t)\end{cases}
$$

With notation $(x)^{+}=\max \{0, x\}, L_{\left[K_{a}, K_{d}\right]}(t)$ in $(2.1)$ can be rewritten as

$$
L_{\left[K_{a}, K_{d}\right]}(t)=\left(Z(t)-K_{a}\right)^{+}-\left(Z(t)-K_{d}\right)^{+}
$$

One sees that $L_{\left[K_{a}, K_{d}\right]}(t)$ can be depicted as a function of $Z(t)$ as shown in Figure 2.

Let $P A Y_{\text {sell } \rightarrow \text { buy }}\left(\tau_{k}\right)$ be the amount to be paid to the protection buyer by the protection seller at time $\tau_{k}, k=0,1, \cdots, K$. This amount is equal to the increment of the cumulative aggregate loss since time $\tau_{k-1}$, that is,

$$
P A Y_{\text {sell } \rightarrow \text { buy }}\left(\tau_{k}\right)=L_{\left[K_{a}, K_{d}\right]}\left(\tau_{k}\right)-L_{\left[K_{a}, K_{d}\right]}\left(\tau_{k-1}\right), \quad k=1,2, \cdots, K
$$

In return, the protection buyer pays to the protection seller by the amount of the unit premium $c_{\left[K_{a}, K_{d}\right]}$ applied to the remaining hedge interval, i.e. $\left(K_{d}-K_{a}\right)$ minus the cumulative 


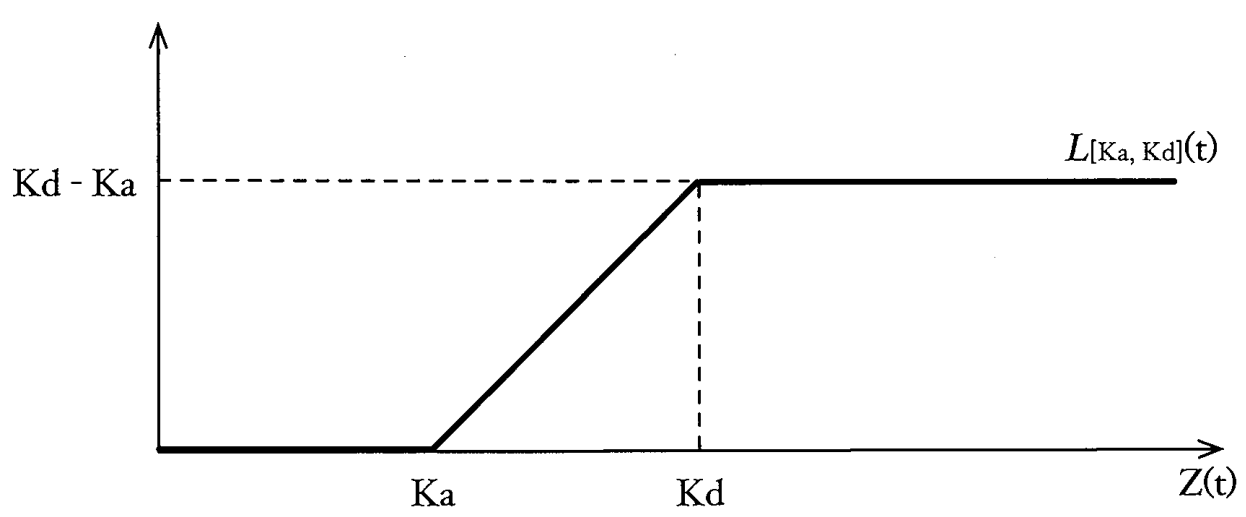

Figure 2: Cumulative loss $L_{\left[K_{a}, K_{d}\right]}$ of a CDO tranche

payment made by the protection seller to the protection buyer up to time $\tau_{k}$. This amount, denoted by $P A Y_{\text {buy } \rightarrow \text { sell }}\left(c_{\left[K_{a}, K_{d}\right]}, \tau_{k}\right)$, can be written as

$$
P A Y_{\text {buy } \rightarrow \text { sell }}\left(c_{\left[K_{a}, K_{d}\right]}, \tau_{k}\right)=c_{\left[K_{a}, K_{d}\right]}\left\{\left(K_{d}-K_{a}\right)-\sum_{j=1}^{k} P A Y_{\text {sell } \rightarrow \text { buy }}\left(\tau_{j}\right)\right\}
$$

for $k=1,2, \cdots, K$. Substitution of (2.3) into (2.4) then yields

$$
P A Y_{\text {buy } \rightarrow \text { sell }}\left(c_{\left[K_{a}, K_{d}\right]}, \tau_{k}\right)=c_{\left[K_{a}, K_{d}\right]}\left\{\left(K_{d}-K_{a}\right)-L_{\left[K_{a}, K_{d}\right]}\left(\tau_{k}\right)\right\} .
$$

Figure 3 illustrates the procedural details discussed above.

In order to satisfy no-arbitrage condition of the credit derivatives market, the unit premium $c_{\left[K_{a}, K_{d}\right]}$ should be set in such a way that the expected payment throughout the contract period from the protection buyer to the protection seller is equal to that from the protection seller to the protection buyer. Let $c_{\left[K_{a}, K_{d}\right]}^{*}$ be this equilibrium premium. One then sees that,

$$
\sum_{k=1}^{K} e^{-r_{f} \tau_{k}} \mathrm{E}\left[P A Y_{\text {sell } \rightarrow \text { buy }}\left(\tau_{k}\right)\right]=\sum_{k=1}^{K} e^{-r_{f} \tau_{k}} \mathrm{E}\left[P A Y_{\text {buy } \rightarrow \text { sell }}\left(c_{\left[K_{a}, K_{d}\right]}^{*}, \tau_{k}\right)\right],
$$

where $r_{f}$ is the risk free interest rate. Here, it is assumed that there exists a risk neutral martingale measure under which all price processes discounted by the interest rate $r_{f}$ are martingales. With an additional assumption that $r_{f}$ is constant, all expectations are taken in this paper with respect to this measure.

By substituting (2.3) and (2.5) into (2.6), it can be solved for $c_{\left[K_{a}, K_{d}\right]}^{*}$ as

$$
c_{\left[K_{a}, K_{d}\right]}^{*}=\frac{\sum_{k=1}^{K} e^{-r_{f} \tau_{k}} \mathrm{E}\left[L_{\left[K_{a}, K_{d}\right]}\left(\tau_{k}\right)-L_{\left[K_{a}, K_{d}\right]}\left(\tau_{k-1}\right)\right]}{\sum_{k=1}^{K} e^{-r_{f} \tau_{k} \mathrm{E}}\left[\left(K_{a}-K_{d}\right)-L_{\left[K_{a}, K_{d}\right]}\left(\tau_{k}\right)\right]},
$$

so that

$$
c_{\left[K_{a}, K_{d}\right]}^{*}=\frac{\sum_{k=1}^{K} e^{-r_{f} \tau_{k}}\left\{\mathrm{E}\left[L_{\left[K_{a}, K_{d}\right]}\left(\tau_{k}\right)\right]-\mathrm{E}\left[L_{\left[K_{a}, K_{d}\right]}\left(\tau_{k-1}\right)\right]\right\}}{\sum_{k=1}^{K} e^{-r_{f} \tau_{k}}\left\{\left(K_{a}-K_{d}\right)-\mathrm{E}\left[L_{\left[K_{a}, K_{d}\right]}\left(\tau_{k}\right)\right]\right\}} .
$$




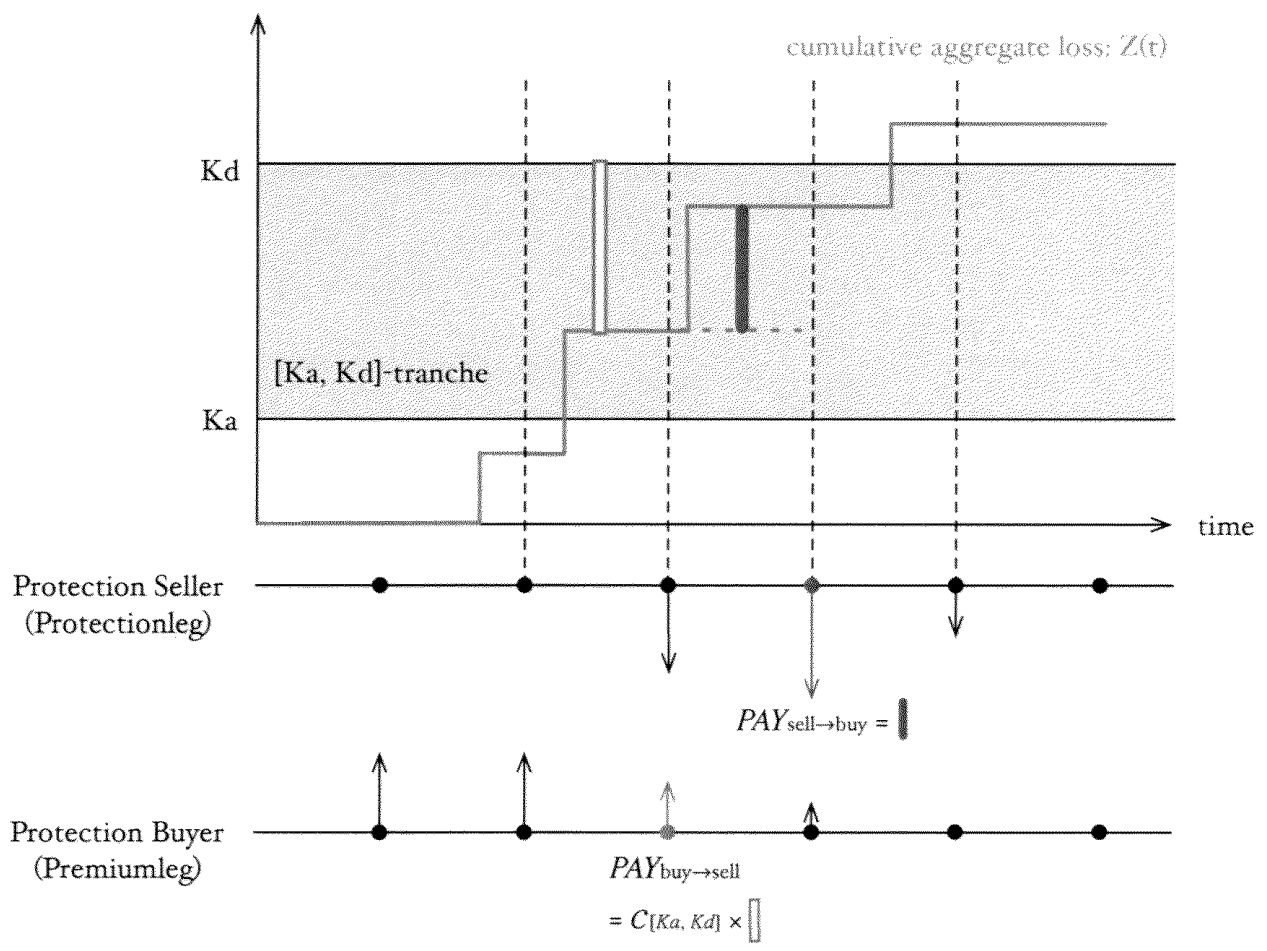

Figure 3: Typical cash flow of a CDO tranche contract

As we will see, by specifying how corporate defaults are generated stochastically and what would be the random nature of the bad debt resulting from such corporate defaults, $Z(t)$ can be modeled as a stochastic process. If the survival function $\bar{F}_{Z}(z, t)=\mathrm{P}[Z(t)>z]$ of $Z(t)$ is obtained, then the expectation of $L_{\left[K_{a}, K_{d}\right]}(t)$ can be evaluated from (2.2) as

$$
\mathrm{E}\left[L_{\left[K_{a}, K_{d}\right]}(t)\right]=\int_{K_{a}}^{\infty} \bar{F}_{Z}(z, t) d z-\int_{K_{d}}^{\infty} \bar{F}_{Z}(z, t) d z+K_{a} \bar{F}_{Z}\left(K_{d}, t\right) .
$$

Consequently, the equilibrium premium $c_{\left[K_{a}, K_{d}\right]}^{*}$ can be computed based on (2.8). In the next section, we employ a semi-Markov modulated Poisson process, which is a special case of the unified multivariate counting process of Sumita and Huang [15], for characterizing occurrences of corporate defaults. The corresponding univariate version of the reward process discussed by Huang and Sumita [6] then characterizes $Z(t)$, yielding the Laplace transform of $Z(t)$ for each $t>0$ based on the results from [6]. The inversion of the Laplace transform can be obtained via the Laguerre transform method, thereby enabling one to assess $c_{\left[K_{a}, K_{d}\right]}^{*}$ numerically.

\section{Corporate Default Structure based on Semi-Markov Modulated Poisson Pro- cess and Associated Cumulative Aggregate Loss Process}

For the CDO pricing problem discussed in the previous section, we assume that corporate defaults are generated by a semi-Markov modulated Poisson process. More specifically, let $J(t)$ be a semi-Markov process on $\mathcal{J}=\{0,1,2\}$, describing the macro-economic condition. Here, state 1 corresponds to the normal economic condition while state 0 and state 2 represent the bad economic condition and the good economic condition respectively. Corporate defaults occur according to a Poisson process $M_{i}(t)$ with intensity $\lambda_{i}$ whenever the semiMarkov process is in state $i$. The $d$-th corporate default in state $i$ results in the random bad 
debt of $Y_{M: i: d}$, where $Y_{M: i: d}$ 's constitute a sequence of i.i.d. random variables with respect to $d$ for each $i$, and such i.i.d. sequences are mutually independent with respect to $i$.

In order to facilitate our analysis, it is assumed that the semi-Markov process $J(t)$ is governed by a matrix cumulative distribution function (c.d.f.) $\underline{\underline{A}}(x)=\left[A_{i j}(x)\right]$, which is absolutely continuous with the matrix probability density function (p.d.f.) $\underline{\underline{a}}(x)=\left[a_{i j}(x)\right]=$ $\frac{d}{d x} \underline{\underline{A}}(x)$. It should be noted that, if we define $A_{i}(x)$ and $\bar{A}_{i}(x)$ by

$$
A_{i}(x)=\sum_{j \in \mathcal{J}} A_{i j}(x) ; \quad \bar{A}_{i}(x)=1-A_{i}(x),
$$

then $A_{i}(x)$ is the c.d.f. and $\bar{A}_{i}(x)$ is the corresponding survival function of the dwell time of $J(t)$ in state $i$. The hazard rate functions associated with the semi-Markov process are then defined as $\eta_{i j}(x)=\frac{a_{i j}(x)}{\bar{A}_{i}(x)}, i, j \in \mathcal{J}$. The Laplace transform of $\underline{\underline{a}}(x)$ is denoted by $\underline{\underline{\alpha}}(w)=\int_{0}^{\infty} e^{-w x} \underline{\underline{a}}(x) d x$.

For notational convenience, the transition epochs of the semi-Markov process are denoted by $\tau_{n}, n \geq 0$, with $\tau_{0}=0$. The age process $X(t)$ associated with the semi-Markov process is then defined as $X(t)=t-\max \left\{\tau_{n}: 0 \leq \tau_{n} \leq t\right\}$. For the cumulative arrival intensity function $L_{i}(x)$ in state $i$, if we consider an age-dependent intensity function $\lambda_{i}(x)$, one has $L_{i}(x)=\int_{0}^{x} \lambda_{i}(y) d y$. The probability of observing $k$ arrivals in state $i$ within the current age of $x$ can then be obtained as

$$
g_{i}(x, k)=e^{-L_{i}(x)} \frac{L_{i}(x)^{k}}{k !}, \quad k=0,1,2, \cdots, \quad i \in \mathcal{J}
$$

Huang and Sumita $[6]$ provide a multivariate reward process $\underline{Z}(t)=\left[Z_{1}(t), Z_{2}(t), \cdots, Z_{K}(t)\right]^{\top}$ defined on $\mathbb{R}^{K}$ given by

$$
\underline{Z}(t)=\int_{0}^{t} \underline{\rho}(J(\tau)) d \tau+\sum_{i \in \mathcal{J}} \sum_{d=1}^{M_{i}(t)} \underline{Y}_{M: i: d}+\sum_{i \in \mathcal{J}} \sum_{j \in \mathcal{J}} \sum_{d=1}^{N_{i j}(t)} \underline{Y}_{N: i j: d},
$$

where $\underline{\rho}(i)$ denotes the multivariate reward rate function while the underlying semi-Markov process $J(t)$ is in state $i \in \mathcal{J} . \underline{Y}_{M: i: d}$ and $\underline{Y}_{N: i j: d}$ represent the vector valued random jumps associated with the $d$-th arrival of $M_{i}(t)$ in state $i$ and the $d$-th jump of $N_{i j}(t)$ describing transitions of $J(t)$ from state $i$ to state $j$, respectively.

In this paper, we let $Z(t)$ be the cumulative aggregate loss of the underlying reference portfolio. One then sees that

$$
Z(t)=\sum_{i \in \mathcal{J}} \sum_{d=1}^{M_{i}(t)} Y_{M: i: d}
$$

We note that $Z(t)$ is a univariate version of $\underline{Z}(t)$ analyzed in Huang and Sumita [6], with the following simplifications.

$$
\left\{\begin{array}{l}
\lambda_{i}(x)=\lambda_{i}, \quad \text { for } i \in \mathcal{J} \\
Y_{N: i j: d}=0 \text { with probability } 1 \\
\rho(i)=0 \text { for all } i \in \mathcal{J}
\end{array}\right.
$$

Let $\zeta(r, s)$ be the double Laplace transform of $Z(t)$, i.e.

$$
\zeta(r, s)=\int_{0}^{\infty} e^{-s t} \mathrm{E}\left[e^{-r Z(t)}\right] d t
$$


By setting $\underline{u}=\underline{1}, \underline{\underline{v}}=\underline{\underline{1}}, w=0+$ in Theorem 3.1 of Huang and Sumita [6], and incorporating the simplifications in (3.3), one sees that

$$
\zeta(r, s)=\underline{p}^{\top}(0) \times \sum_{\ell=0}^{\infty} \underline{\underline{\beta}}\left(s \underline{1}, \underline{\theta}_{M}(r)\right)^{\ell} \times \underline{\underline{\beta}}_{D}^{*}\left(s \underline{1}_{M}(r)\right) \times \underline{1},
$$

where $Z(0)=0, \underline{p}(0)$ denotes the initial probability vector of $J(0)$, and $\underline{\theta}_{M}(r)=\left[\theta_{M: 0}(r), \cdots, \theta_{M: J}(r)\right]^{\top}$ with $\theta_{M: i}(r)$ denoting the Laplace transform of distribution of $Y_{M: i: d}$, i.e., given the common probability density function $\mathcal{Y}_{M: i}(z)$ of $Y_{M: i: d}$,

$$
\theta_{M: i}(r)=\int_{0}^{\infty} e^{-r z} \mathcal{Y}_{M: i}(z) d z
$$

The Laplace transform generating function $\underline{\underline{\beta}}(\underline{w}, \underline{u})$ and $\underline{\widehat{\beta}}_{D}^{*}(\underline{w}, \underline{u})$ are defined as

$$
\begin{aligned}
& \underline{\widehat{\beta}}(\underline{w}, \underline{u})=\left[\widehat{\beta}_{i j}\left(w_{i}, u_{i}\right)\right] ; \quad \widehat{\beta}_{i j}\left(w_{i}, u_{i}\right)=\sum_{m_{i}=0}^{\infty}\left(\int_{0}^{\infty} e^{-w_{i} x} a_{i j}(x) g_{i}\left(x, m_{i}\right) d x\right) u_{i}^{m_{i}}, \\
& \underline{\widehat{\beta}}_{D}^{*}(\underline{w}, \underline{u})=\left[\delta_{\{i=j\}} \widehat{\beta}_{i}^{*}\left(w_{i}, u_{i}\right)\right] ; \quad \widehat{\beta}_{i}^{*}\left(w_{i}, u_{i}\right)=\sum_{m_{i}=0}^{\infty}\left(\int_{0}^{\infty} e^{-w_{i} x} \bar{A}_{i}(x) g_{i}\left(x, m_{i}\right) d x\right) u_{i}^{m_{i}} .
\end{aligned}
$$

Given $t>0$, the random variable $Z(t)$ has a mass at the origin denoted by $z_{0}(t)=$ $\mathrm{P}[Z(t)=0]$, and an absolutely continuous density $f_{Z_{+}}(z, t)$ for $z \geq 0$. More formally, $F_{Z}(z, t)=\mathrm{P}[Z(t) \leq z]$ can be written as

$$
F_{Z}(z, t)=z_{0}(t)+\int_{0}^{z} f_{Z_{+}}(x, t) d x
$$

Accordingly, $\zeta(r, s)$ in (3.4) is given by

$$
\zeta(r, s)=\zeta_{0}(s)+\zeta_{+}(r, s)
$$

where

$$
\zeta_{0}(s)=\int_{0}^{\infty} e^{-s t} z_{0}(t) d t ; \quad \zeta_{+}(r, s)=\int_{0}^{\infty} \int_{0}^{\infty} e^{-s t} e^{-r z} f_{Z_{+}}(z, t) d z d t
$$

It can be readily seen that $\zeta_{0}(s)=\lim _{r \rightarrow \infty} \zeta(r, s)$. We note from $(3.6)$ that $\lim _{r \rightarrow \infty} \underline{\theta}_{M}(r)=$ $\underline{0}$. It then follows from Equation (3.5) that

$$
\zeta_{0}(s)=\underline{p}^{\top}(0) \times\left\{\underline{\underline{I}}+\sum_{\ell=1}^{\infty}\left[\alpha_{i j}\left(s+\lambda_{i}\right)\right]_{i, j \in \mathcal{J}}^{\ell}\right\} \times\left[\frac{1-\alpha_{i}\left(s+\lambda_{i}\right)}{s+\lambda_{i}}\right]_{i \in \mathcal{J}} .
$$

From (3.5) together with $(3.12), \zeta_{+}(r, s)=\zeta(r, s)-\zeta_{0}(s)$ can then be obtained, after a little algebra, as

$$
\zeta_{+}(r, s)=\underline{p}^{\top}(0) \times\left\{\underline{\underline{I}}+\sum_{\ell=1}^{\infty} \underline{\widehat{\beta}}_{+}\left(s \underline{1}, \underline{\theta}_{M}(r)\right)^{\ell}\right\} \times \underline{\widehat{\beta}}_{+: D}^{*}\left(s \underline{1}, \underline{\theta}_{M}(r)\right) \times \underline{1},
$$


where

$$
\begin{aligned}
\widehat{\beta}_{+: i j}\left(s, \theta_{M: i}(r)\right) & =\widehat{\beta}_{i j}\left(s, \theta_{M: i}(r)\right)-\alpha_{i j}\left(s+\lambda_{i}\right) \\
& =\sum_{k=1}^{\infty} \theta_{M: i}^{k}(r) \int_{0}^{\infty} e^{-s t} a_{i j}(t) e^{-\lambda_{i} t} \frac{\left(\lambda_{i} t\right)^{k}}{k !} d t
\end{aligned}
$$

and

$$
\begin{aligned}
\widehat{\beta}_{+: i}^{*}\left(s, \theta_{M: i}(r)\right) & =\widehat{\beta}_{i}^{*}\left(s, \theta_{M: i}(r)\right)-\frac{1-\alpha_{i}\left(s+\lambda_{i}\right)}{s+\lambda_{i}} \\
& =\sum_{k=1}^{\infty} \theta_{M: i}^{k}(r) \int_{0}^{\infty} e^{-s t} \bar{A}_{i}(t) e^{-\lambda_{i} t} \frac{\left(\lambda_{i} t\right)^{k}}{k !} d t
\end{aligned}
$$

\section{Development of Numerical Algorithms for Evaluating the Distribution of $Z(t)$ via the Laguerre Transform}

In this section, we develop numerical algorithms for evaluating $z_{0}(t)$ and $f_{Z_{+}}(z, t)$ via the Laguerre transform based on (3.12) through (3.15). A succinct summary of the Laguerre transform is provided in Appendix A. The reader is referred to Sumita and Kijima [17, 18] for further details concerning the algorithmic aspects of the Laguerre transform.

Let

$$
\begin{aligned}
& a_{k: i j}(t)=a_{i j}(t) e^{-\lambda_{i} t} \frac{\left(\lambda_{i} t\right)^{k}}{k !}, \quad k=0,1,2, \cdots \\
& \bar{A}_{k: i}(t)=\bar{A}_{i}(t) e^{-\lambda_{i} t} \frac{\left(\lambda_{i} t\right)^{k}}{k !}, \quad k=0,1,2, \cdots .
\end{aligned}
$$

For notational simplicity, we define

$$
\begin{aligned}
& \left\{a_{n}\right\}_{n: N}=\left\{a_{0}, a_{1}, \cdots, a_{N}\right\} \\
& \left\{a_{(m, n)}\right\}_{m n: M N}=\left\{a_{(0,0)}, a_{(0,1)}, \cdots, a_{(0, N)}, \cdots, a_{(M, 0)}, a_{(M, 1)}, \cdots, a_{(M, N)}\right\}
\end{aligned}
$$

where $a_{n}$ or $a_{(m, n)}$ may be a number, a vector or a matrix, and $M$ or $N$ denotes a positive integer or $\infty$. With this notation, we define the corresponding Laguerre sharp and dagger coefficients $\left\{\underline{\underline{a}}_{k: n}^{\#}\right\}_{n: \infty},\left\{\underline{a}_{k: n}^{\dagger}\right\}_{n: \infty},\left\{\underline{A}_{k: n}^{\#}\right\}_{n: \infty}$ and $\left\{\underline{A}_{k: n}^{\dagger}\right\}_{n: \infty}$, i.e.

$$
\begin{aligned}
& \underline{\underline{T}}_{a_{k}}^{\#}(u)=\left.\int_{0}^{\infty} e^{-s t} \underline{\underline{a}}_{k}(t) d t\right|_{s=\frac{1}{2} \frac{1+u}{1-u}}=\sum_{n=0}^{\infty} \underline{\underline{a}}_{k: n}^{\#} u^{n}, \\
& \underline{T}_{\bar{A}_{k}}^{\#}(u)=\left.\int_{0}^{\infty} e^{-s t} \underline{\bar{A}}_{k}(t) d t\right|_{s=\frac{1}{2} \frac{1+u}{1-u}}=\sum_{n=0}^{\infty} \underline{\bar{A}}_{k: n}^{\#} u^{n},
\end{aligned}
$$

and $\underline{\underline{a}}_{k: n}^{\dagger}=\sum_{m=0}^{n} \underline{\underline{a}}_{k: m}^{\#}, \underline{\bar{A}}_{k: n}^{\dagger}=\sum_{m=0}^{n} \underline{\bar{A}}_{k: m}^{\#}$.

Similarly, for $\theta_{M: i}(r)=\int_{0}^{\infty} e^{-r z} \mathcal{Y}_{M: i}(z) d z$, the Laguerre coefficients $\left\{\underline{y}_{m}^{\#}\right\}_{m: \infty}$ and $\left\{\underline{y}_{m}^{\dagger}\right\}_{m: \infty}$ are defined as

$$
\underline{T}_{\mathcal{Y}}^{\#}(v)=\left.\underline{\theta}_{M}(r)\right|_{r=\frac{1}{2} \frac{1+v}{1-v}}=\sum_{m=0}^{\infty} \underline{y}_{m}^{\#} v^{m}
$$


and $\underline{y}_{m}^{\dagger}=\sum_{n=0}^{m} \underline{y}_{n}^{\#}$. We note that the Laguerre transform generating functions associated with time $t$ are expressed using $u$ with index $n$, while those corresponding to the reward $z$ are described in terms of $v$ with index $m$.

By using the array operation CONV, VCONV, VBCONV, VMCONV and VMBCONV defined in Appendix B, one has

$$
\begin{aligned}
& \left\{\underline{\pi}_{0: n}^{\#}(1)\right\}_{n: \infty} \stackrel{\text { def }}{\Leftrightarrow} \underline{\pi}_{0: n}^{\# \top}(1)=\underline{p}^{\top}(0) \cdot \underline{a}_{0: n}^{\#}, \quad n=0,1, \cdots, \\
& \left\{\underline{\pi}_{0: n}^{\#}(\ell)\right\}_{n: \infty}=\operatorname{VMCONV}\left(\left\{\underline{\pi}_{0: n}^{\#}(\ell-1)\right\}_{n: \infty},\left\{\underline{\underline{a}}_{0: n}^{\#}\right\}_{n: \infty}\right), \quad \ell=2,3, \cdots, \\
& \left\{\underline{\pi}_{0: n}^{\#}\right\}_{n: \infty} \stackrel{\text { def }}{\Leftrightarrow} \underline{\pi}_{0: n}^{\#}=\sum_{\ell=1}^{\infty} \underline{\pi}_{0: n}^{\#}(\ell), \quad n=0,1, \cdots, \\
& \left\{\Pi_{0: n}^{\#}\right\}_{n: \infty} \stackrel{\text { def }}{\Leftrightarrow} \Pi_{0: n}^{\#}=\underline{p}^{\top}(0) \cdot \underline{\bar{A}}_{0: n}^{\#}, \quad n=0,1, \cdots .
\end{aligned}
$$

From Equation (3.12), the Laguerre sharp coefficients $\left\{z_{0: n}^{\#}\right\}_{n: \infty}$ of $z_{0}(t)$ is then obtained as

$$
\left\{z_{0: n}^{\#}\right\}_{n: \infty}=\left\{\Pi_{0: n}^{\#}\right\}_{n: \infty}+\operatorname{VCONV}\left(\left\{\underline{\pi}_{0: n}^{\#}\right\}_{n: \infty},\left\{\underline{A}_{0: n}^{\#}\right\}_{n: \infty}\right)
$$

For $f_{Z_{+}}(z, t)$, let $\left\{y_{i: m}^{\#}(k)\right\}_{m: \infty}$ be defined by

$$
\left\{y_{i: m}^{\#}(k)\right\}_{m: \infty}=\operatorname{CONV}\left(\left\{y_{i: m}^{\#}(k-1)\right\}_{m: \infty},\left\{y_{i: m}^{\#}\right\}_{m: \infty}\right), \quad k=2,3, \cdots,
$$

with $\left\{y_{i: m}^{\#}(1)\right\}_{m: \infty}=\left\{y_{i: m}^{\#}\right\}_{m: \infty}$ for $i \in \mathcal{J}$. We also define

$$
\begin{aligned}
& \left\{b_{+: i j:(m, n)}^{\dagger}\right\}_{m n: \infty \infty} \stackrel{\text { def }}{\Leftrightarrow} b_{+: i j:(m, n)}^{\dagger}=\sum_{k=1}^{\infty} y_{i: m}^{\dagger}(k) \times a_{k: i j: n}^{\dagger}, \\
& \left\{b_{+: i:(m, n)}^{* \dagger}\right\}_{m n: \infty \infty} \stackrel{\text { def }}{\Leftrightarrow} b_{+: i:(m, n)}^{* \dagger}=\sum_{k=1}^{\infty} y_{i: m}^{\dagger}(k) \times \bar{A}_{k: i: n}^{\dagger} .
\end{aligned}
$$

Correspondingly, $\left\{b_{+: i j:(m, n)}^{\#}\right\}_{m n: \infty \infty}$ and $\left\{b_{+: i:(m, n)}^{* \#}\right\}_{m n: \infty \infty}$ can be given by

$$
\begin{aligned}
& b_{+: i j:(m, n)}^{\#}=b_{+: i j:(m, n)}^{\dagger}-b_{+: i j:(m-1, n)}^{\dagger}-b_{+: i j:(m, n-1)}^{\dagger}+b_{+: i j:(m-1, n-1)}^{\dagger}, \\
& b_{+: i:(m, n)}^{* \#}=b_{+: i:(m, n)}^{* \dagger}-b_{+: i:(m-1, n)}^{* \dagger}-b_{+: i:(m, n-1)}^{* \dagger}+b_{+: i:(m-1, n-1)}^{* \dagger} .
\end{aligned}
$$

This then yields the matrix sequence $\left\{\underline{\underline{b}}_{+:(m, n)}^{\#}\right\}_{m n: \infty \infty}$ with $\underline{\underline{b}}_{+:(m, n)}^{\#}=\left[b_{+: i j:(m, n)}^{\#}\right]$ and the vector sequence $\left\{\underline{b}_{+:(m, n)}^{* \#}\right\}_{m n: \infty \infty}$ with $\underline{b}_{+:(m, n)}^{* \#}=\left[b_{+: i:(m, n)}^{* \#}\right]_{i \in \mathcal{J}}$.

Using these matrix and vector sequences, we define

$$
\begin{aligned}
& \left\{\underline{\pi}_{+:(m, n)}^{\#}(1)\right\}_{m n: \infty} \stackrel{\text { def }}{\Leftrightarrow} \underline{\pi}_{+:(m, n)}^{\# \top}(1)=\underline{p}^{\top}(0) \cdot \underline{\underline{b}}_{+:(m, n)}^{\#}, \quad m, n=0,1, \cdots, \\
& \left\{\underline{\pi}_{+:(m, n)}^{\#}(\ell)\right\}_{m n: \infty \infty}=\operatorname{VMBCONV}\left(\left\{\underline{\pi}_{+:(m, n)}^{\#}(\ell-1)\right\}_{m n: \infty \infty},\left\{\underline{b}_{+:(m, n)}^{\#}\right\}_{m n: \infty \infty}\right) \text {, } \\
& \ell=2,3, \cdots \\
& \left\{\underline{\pi}_{+:(m, n)}^{\#}\right\}_{m n: \infty \infty} \stackrel{\text { def }}{\Leftrightarrow} \underline{\pi}_{+:(m, n)}^{\#}=\sum_{\ell=1}^{\infty} \underline{\pi}_{+:(m, n)}^{\#}(\ell), \quad m, n=0,1, \cdots, \\
& \left\{\Pi_{+:(m, n)}^{\#}\right\}_{m n: \infty \infty} \stackrel{\text { def }}{\Leftrightarrow} \Pi_{+:(m, n)}^{\#}=\underline{p}^{\top}(0) \cdot \underline{b}_{+:(m, n)}^{* \#}, \quad m, n=0,1, \cdots .
\end{aligned}
$$


From Equation (3.13), the Laguerre sharp coefficients $\left\{z_{+:(m, n)}^{\#}\right\}_{m n: \infty \infty}$ of $f_{Z_{+}}(z, t)$ can then be obtained as

$$
\begin{aligned}
\left\{z_{+:(m, n)}^{\#}\right\}_{m n: \infty \infty}= & \left\{\Pi_{+:(m, n)}^{\#}\right\}_{m n: \infty \infty} \\
& +\operatorname{VBCONV}\left(\left\{\underline{\pi}_{+:(m, n)}^{\#}\right\}_{m n: \infty \infty},\left\{\underline{b}_{+:(m, n)}^{* \#}\right\}_{m n: \infty \infty}\right) .
\end{aligned}
$$

Consequently, $z_{0}(t)$ and $f_{Z_{+}}(z, t)$ can be evaluated as

$$
\begin{aligned}
& z_{0}(t)=\sum_{n=0}^{\infty} z_{0: n}^{\dagger} \ell_{n}(t) \\
& f_{Z_{+}}(z, t)=\sum_{n=0}^{\infty} \sum_{m=0}^{\infty} z_{+:(m, n)}^{\dagger} \ell_{m n}(z, t)
\end{aligned}
$$

where $z_{0: n}^{\dagger}=\sum_{i=0}^{n} z_{0: i}^{\#}, z_{+:(m, n)}^{\dagger}=\sum_{j=0}^{n} \sum_{i=0}^{m} z_{+:(i, j)}^{\#}$ and $\ell_{m n}(z, t)=\ell_{m}(z) \ell_{n}(t)$ with $\ell_{n}(x)=$ $\frac{e^{x / 2}}{n !}\left(\frac{d}{d x}\right)^{n}\left(x^{n} e^{-x}\right)$ for $n=0,1,2, \cdots$.

The Laguerre coefficients of an exponential density function can be expressed in a closed form as shown in Appendix A. For probability density functions that are generated from exponential density functions through mixings and/or convolutions, their Laguerre coefficients can be obtained by employing operational properties of the Laguerre transform. For example, let $C M_{m}$ be a class of completely monotone density functions defined by

$$
C M_{m}=\left\{f: \varphi_{f}(s)=\sum_{i=1}^{m} p_{i} \frac{\lambda_{i}}{s+\lambda_{i}}, p_{i} \geq 0, \sum_{i=1}^{m} p_{i}=1\right\}
$$

where $\varphi_{f}(s)=\int_{0}^{\infty} e^{-s t} f(t) d t$. We also introduce a class of Polya Frequency functions of order $n$ denoted by $P F_{n}$, where

$$
P F_{n}=\left\{f: \varphi_{f}(s)=\prod_{i=1}^{n} \frac{\lambda_{i}}{s+\lambda_{i}}\right\}
$$

The Laguerre transform of any probability density function in $C M_{m} \cup P F_{n}$ can be readily obtained through mixings and/or convolutions of Laguerre transforms of exponential density functions. Accordingly, if $a_{i j}(x) \in C M_{m} \cup P F_{n}$, the corresponding $z_{0}(t)$ and $f_{Z_{+}}(z, t)$ can be evaluated based on the algorithmic procedures given in this section. It is worth noting that a family of $P F_{n}$ 's for $n \in \mathbb{N}$ is dense in the class of absolutely continuous probability density functions associated with non-negative random variables.

\section{Numerical Results}

The purpose of this section is to demonstrate the power of the numerical algorithms developed in the previous section. In practice, it is natural to assume that the economic condition changes in a lattice continuous manner so that $J(t)$ becomes a skip-free semiMarkov process. We also assume that the dwell time of $J(t)$ in each state belongs to $P F_{2}$. More specifically, the matrix Laplace transform $\underline{\underline{\alpha}}(s)$ of the semi-Markov matrix of $J(t)$ is given by

$$
\underline{\underline{\alpha}}(s)=\left[p_{i j} \alpha_{i}(s)\right]
$$


where $p_{i j}=0$ if $i=j$ or $|i-j|>1$ and $p_{i j}>0$ otherwise, $\sum_{j \in \mathcal{J}} p_{i j}=1$, and

$$
\alpha_{i}(s)=\int_{0}^{\infty} e^{-s t} d A_{i}(t)=\frac{\alpha_{i, 1}}{\alpha_{i, 1}+s} \cdot \frac{\alpha_{i, 2}}{\alpha_{i, 2}+s}, \quad i \in \mathcal{J} .
$$

The amount of the bad debt generated by each default is assumed to be exponentially distributed with mean $\mu^{-1}$. Specific parameter values to be employed in this setting are summarized in Table 1.

Table 1: Underlying parameter values for evaluating $z_{0}(t)$ and $f_{Z_{+}}(z, t)$

\begin{tabular}{cc|cc|cc}
\hline Parameter & Value & Parameter & Value & Parameter & Value \\
\hline $\mathcal{J}$ & $\{0,1,2\}$ & $L$ & 30 & $K$ & 20 \\
$\underline{\lambda}$ & {$[1.2,1,0.8]^{\top}$} & $\underline{\alpha}_{\bullet, 1}$ & {$[1,1.2,1.4]^{\top}$} & $\underline{\alpha}_{\bullet, 2}$ & {$[2,1.8,1.6]^{\top}$} \\
& & & & & $\underline{p}$ \\
$\mu$ & 1 & $\underline{p}(0)$ & {$[0,1,0]^{\top}$} & {$\left[\begin{array}{ccc}0 & 1 & 0 \\
0.6 & 0 & 0.4 \\
0 & 1 & 0\end{array}\right]$} \\
\hline
\end{tabular}

The values of $z_{0}(t)$ and $f_{Z_{+}}(z, t)$ are depicted in Figures 4 and 5 respectively. The corresponding survival function $\bar{F}_{Z}(z, t)$ is shown in Figure 6 . We note that the survival function $\bar{F}_{Z}(0, t)$ has jumps of size $z_{0}(t)$ at $Z(t)=0$, which diminishes as $t \rightarrow \infty$.

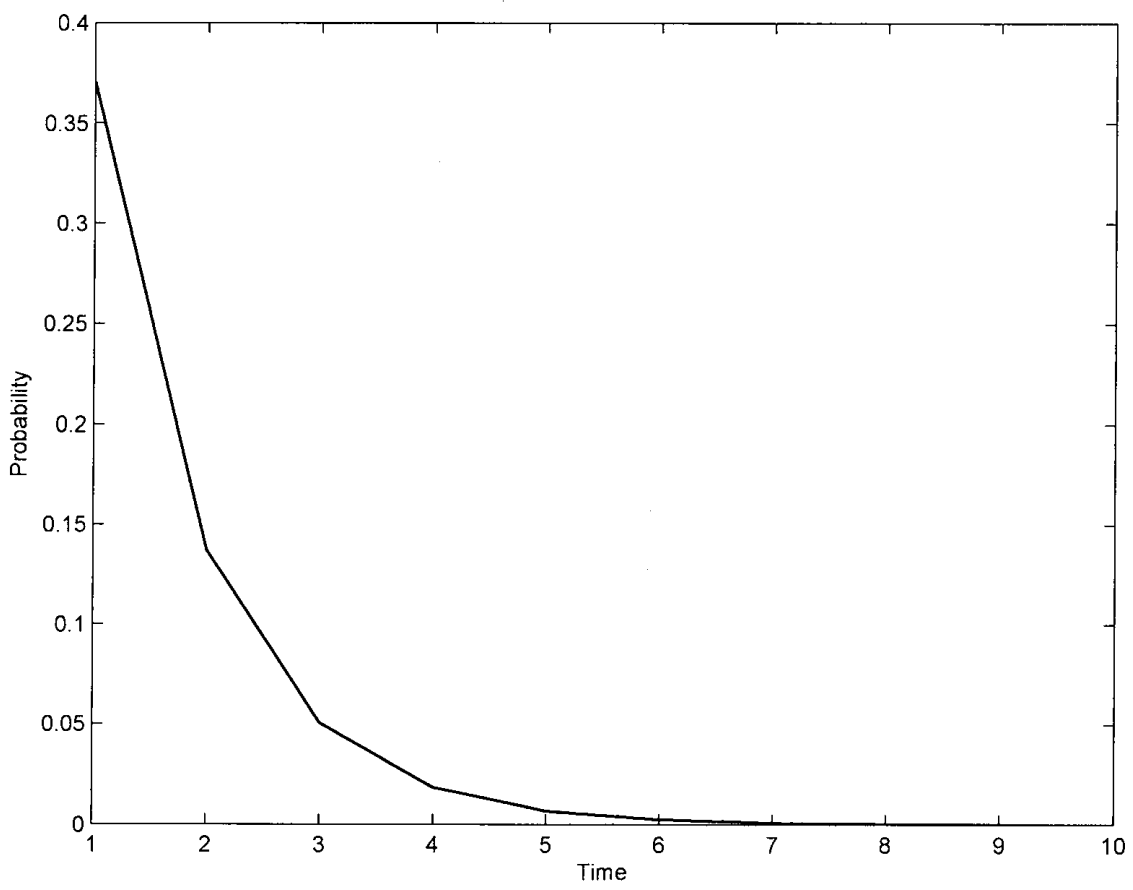

Figure 4: Probability of $z_{0}(t)$

In Figure 7, the asymptotic expansion of $\frac{E[Z(t)]}{t}$ as $t \rightarrow \infty$ is compared with the exact value of $\frac{\mathrm{E}[Z(t)]}{t}$ obtained through the Laguerre transform procedure. In this numerical 


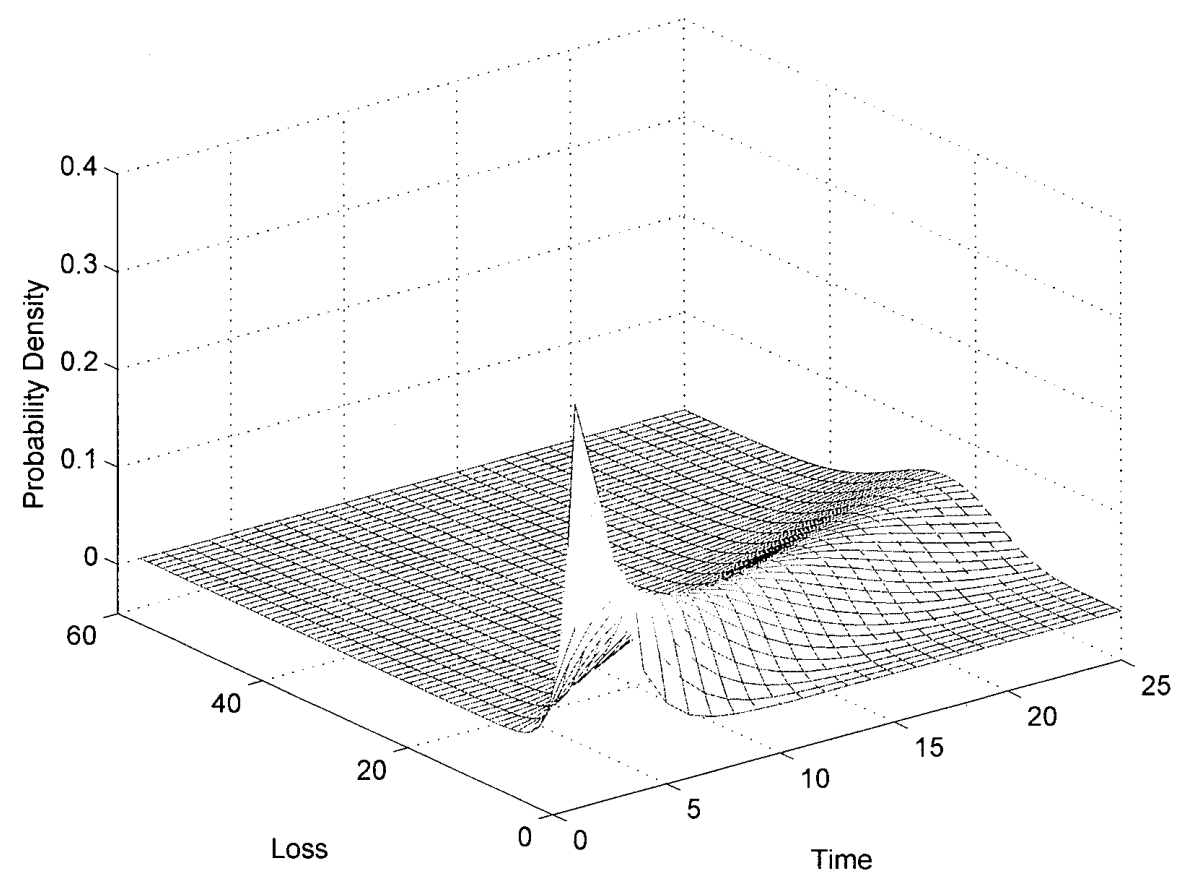

Figure 5: Probability density of $f_{Z_{+}}(z, t)$

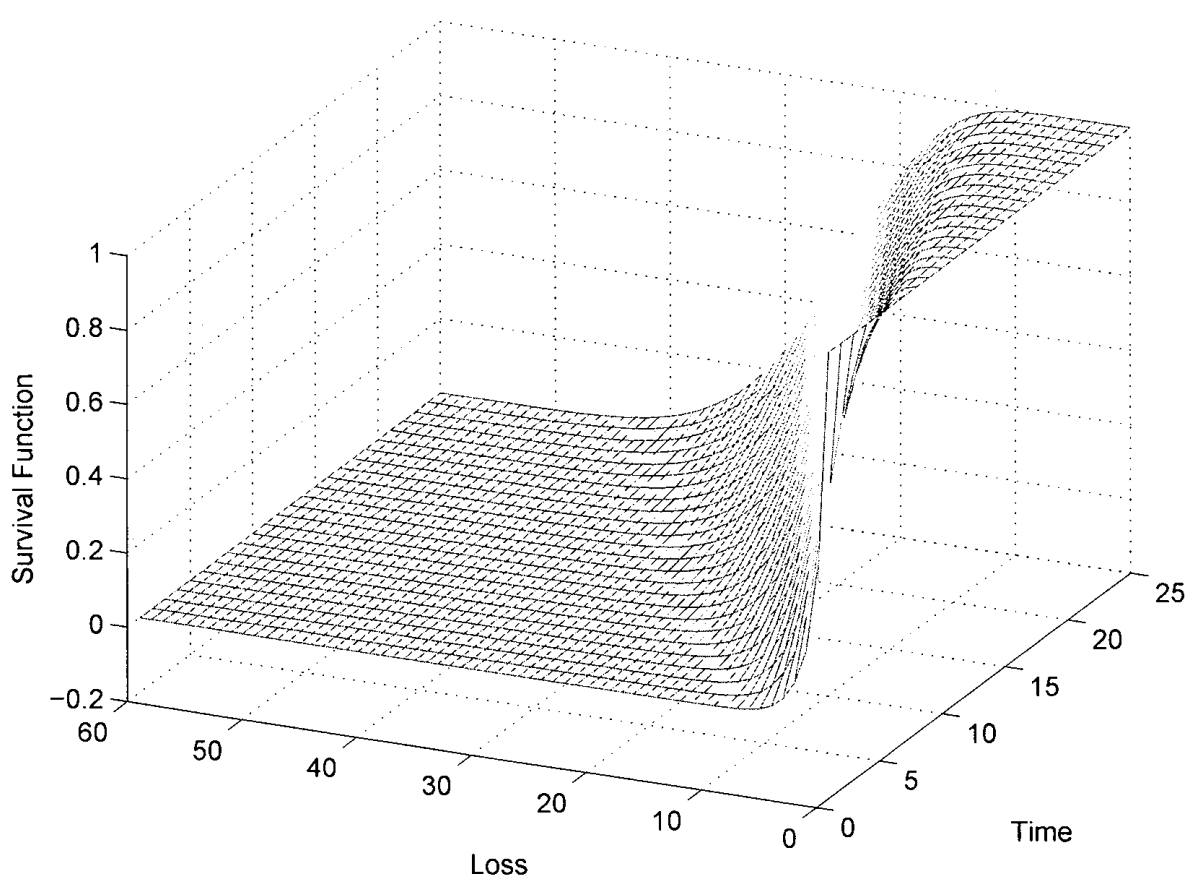

Figure 6: Survival function $\bar{F}_{Z}(z, t)$ 
example, the asymptotic expansion is rather slow, demonstrating the importance of the Laguerre transform procedure when $t$ is not sufficiently large.

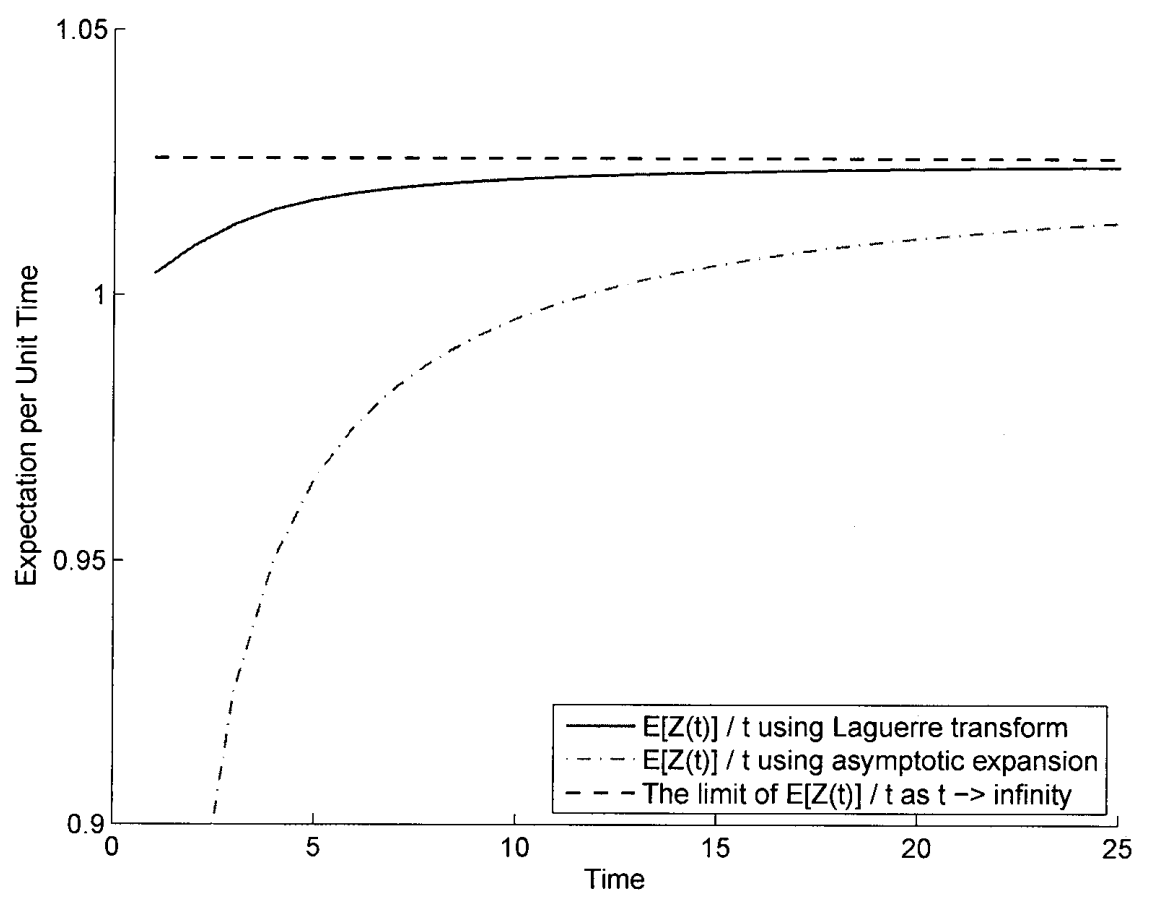

Figure 7: Convergence of $\frac{\mathrm{E}[Z(t)]}{t}$

For the CDO pricing problem discussed in Section 2, the equilibrium unit premium $c_{\left[K_{a}, K_{d}\right]}^{*}$ in Equation (2.8) can be obtained by using Equation (2.9). The algorithmic procedure for evaluating $c_{\left[K_{a}, K_{d}\right]}^{*}$ is also shown in Appendix B. Let the risk free interest rate be $r_{f}=0.03$, and let the maturity be 5 years. There are 4 periods in each year. In this setting, the equilibrium unit premiums for various tranches are given in Table 2 . In the first block of Table 2, it can be seen that the underlying risk for the protection seller decreases as the tranche interval shifts upwards, where the equilibrium unit premium decreases from $12.27 \%$ for $[10,20]$ to $1.30 \mathrm{bp}$ for $[40,50]$. In the second block, $K_{a}$ is fixed at $K_{a}=10$ while $K_{d}$ is changed from 20 to 50 . The underlying risk for the protection seller also decreases as $K_{d}$ increases, where the equilibrium unit premium decreases from $12.27 \%$ for $[10,20]$ to $1.55 \%$ for $[10,50]$. In the third block, $K_{a}$ is increased from 10 to 40 while $K_{d}$ is fixed at $K_{d}=50$. The underlying risk for the protection seller again decreases along the direction of this change, where the equilibrium unit premium decreases from $1.55 \%$ for $[10,50]$ to 1.30 bp for $[40,50]$. These results suggest the following.

1. The risk of the protection seller for the CDO model decreases as a tranche of the same width shifts upward.

2. The above risk also decreases as the detachment point $K_{d}$ increases while the attachment point $K_{a}$ is fixed.

3. A similar phenomenon to 2 is observed when the attachment point $K_{a}$ increases while the detachment point $K_{d}$ is fixed. 
Table 2: Numerical results of equilibrium unit premium of CDO's

\begin{tabular}{ccccc}
\hline$\left[K_{a}, K_{d}\right]$ & {$[10,20]$} & {$[20,30]$} & {$[30,40]$} & {$[40,50]$} \\
\hline Unit premium & $12.27 \%$ & $2.60 \%$ & $27.03 \mathrm{bp}$ & $1.30 \mathrm{bp}$ \\
\hline \multicolumn{5}{c}{} \\
\hline$\left.K_{a}, K_{d}\right]$ & {$[10,20]$} & {$[10,30]$} & {$[10,40]$} & {$[10,50]$} \\
\hline Unit premium & $12.27 \%$ & $3.66 \%$ & $2.14 \%$ & $1.55 \%$ \\
\hline \multicolumn{5}{c}{} \\
\hline$\left[K_{a}, K_{d}\right]$ & {$[10,50]$} & {$[20,50]$} & {$[30,50]$} & {$[40,50]$} \\
\hline Unit premium & $1.55 \%$ & $54.16 \mathrm{bp}$ & $9.36 \mathrm{bp}$ & $1.30 \mathrm{bp}$ \\
\hline "bp" = basis point, i.e. $1 \mathrm{bp}=0.01 \%$
\end{tabular}

\section{Concluding Remarks}

In this paper, a dynamic model is developed for assessing the price of a CDO tranche. A cumulative random shock process generated from a semi-Markov modulated Poisson process is employed to capture the stochastic behavior of the cumulative aggregate loss of the underlying reference portfolio. By using the transform results obtained in Huang and Sumita [6] together with the Laguerre transform method developed by Keilson and Nunn [7], Keilson, Nunn and Sumita [8], and further studied by Sumita [13], computational algorithms are developed for evaluating the distribution of the loss process for the underlying CDO model, which in turn enables one to evaluate the price of the CDO tranche. Some numerical results are presented, demonstrating the efficiency of the algorithms.

In order to calibrate the reality through this model, it is necessary to establish an efficient procedure to estimate the underlying parameters specified in Table 1. These parameters cannot be estimated by collecting historical data of corporate defaults as well as switches of macro economic conditions. For this purpose, one has to obtain sufficient data concerning CDO tranches as well as the associated prices from the market. The underlying parameters ought to be estimated so as to provide "the minimum distance" to such market data. It is quite difficult to overcome this hurdle without closely working with a financial institution which has direct access to such information.

\section{Acknowledgement}

The authors wish to thank two anonymous referees as well as the editor for providing constructive comments which contributed to improve the original version of the paper. This research is supported by MEXT Grand-in-Aid for Scientific Research (C) 22500082.

\section{References}

[1] M. Arnsdorf and I. Halperin: BSLP: Markovian bivariate spread-loss model for portfolio credit derivatives. Quantitative finance papers, arXiv.org, (2009). URL: http://arxiv .org/pdf/0901.3398.

[2] T.R. Bielecki, A. Vidozzi, and L. Vidozzi: A Markov copulae approach to pricing and hedging of credit derivatives and ratings triggered step-up bonds. The Journal of Credit Risk, 4-1 (2008).

[3] J. de Kock, H. Kraft, and M. Steffensen: CDOs in chains. Wilmott Magazin, (2007).

[4] W. Fischer and K. Meier-Hellstern: The Markov-modulated Poisson process (MMPP) cookbook. Performance Evaluation, 18-2 (1993), 149-171. 
[5] R. Frey and J. Backhaus: Portfolio credit risk models with interacting default intensities: a Markovian approach. preprint, department of mathematics, Universität Leipzig, (2004).

[6] J.-P. Huang and U. Sumita: Dynamic analysis of a multivariate reward process defined on the UMCP with application to optimal preventive maintenance policy problems in manufacturing. Department of Social Systems and Management Discussion Paper Series 1272, University of Tsukuba, (2011).

[7] J. Keilson and W.R. Nunn: Laguerre transform as a tool for the numerical solution of integral equations of convolution type. Applied Mathematics and Computation, 7 (1979), 313-359.

[8] J. Keilson, W.R. Nunn, and U. Sumita: The bilateral Laguerre transform. Applied Mathematics and Computation, 8 (1981), 137-174.

[9] D. Lando: Credit Risk Modeling: Theory and Applications. (Princeton University Press, 2004).

[10] D.X. Li: On default correlation: a copula function approach. Journal of Fixed Income, 9 (2000), 43-54.

[11] P.J. Schönbucher: Portfolio losses and the term structure of loss transition rates: a new methodology for the pricing of portfolio credit derivatives. working paper, ETH Zürich, (2006).

[12] J. Sidenius, V. Piterbarg, and L. Andersen: A new framework for dynamic credit portfolio loss modeling. International Journal of Theoretical and Applied Finance, 112 (2008), 163-197. doi:10.1142/S0219024908004762.

[13] U. Sumita: Development of the Laguerre Transform Method for Numerical Exploration of Applied Probability Models. Ph.d. thesis, William E. Simon Graduate School of Business Administration, University of Rochester, (1981).

[14] U. Sumita: The matrix Laguerre transform. Applied Mathematics and Computation, 15 (1984), 1-28.

[15] U. Sumita and J.-P. Huang: Dynamic analysis of a unified multivariate counting process and its asymptotic behavior. International Journal of Mathematics and Mathematical Sciences, vol.2009 (Article ID 219532, 43pages, 2009). doi:10.1155/2009/219532.

[16] U. Sumita and M. Kijima: The bivariate Laguerre transform and its applications: numerical exploration of bivariate processes. Advances in Applied Probability, 17 (1985), 683-708.

[17] U. Sumita and M. Kijima: Theory and algorithms of the Laguerre transform, part I: theory. Journal of the Operations Research Society of Japan, 31-4 (1988), 467-494.

[18] U. Sumita and M. Kijima: Theory and algorithms of the Laguerre transform, part II: algorithm. Journal of the Operations Research Society of Japan, 34-4 (1991), 449-477.

[19] G. Szegö: Orthogonal Polynomials, volume 23 of American Mathematical Society Colloquium Publications. (American Mathematical Society, 4th edition, 1975).

[20] H. Takada and U. Sumita: Credit risk model with contagious default dependencies affected by macro-economic condition. European Journal of Operational Research, 2142 (2011), 365-379.

[21] H. Takada, U. Sumita, and K. Takahashi: Pricing collateralized debt obligations with Markov-modulated Poisson processes. Quantitative Finance, 11-12 (2011), 1761-1771. 


\section{A. Introduction of the Laguerre Transform Method as a Tool of Numerical Inversion Laplace Transforms}

In this section, the Laguerre transform method for numerical inversion of Laplace transforms is succinctly summarized. We consider the Laguerre polynomials $\mathcal{L}_{n}(x)$ defined by

$$
\mathcal{L}_{n}(x)=\frac{e^{x}}{n !}\left(\frac{d}{d x}\right)^{n}\left(x^{n} e^{-x}\right), \quad n=0,1,2, \cdots
$$

and the associated Laguerre functions

$$
\ell_{n}(x)=e^{-x / 2} \mathcal{L}_{n}(x)
$$

It is known that $\left\{\ell_{n}(x)\right\}$ constitute an orthonormal basis of $L_{2}(0, \infty)=\left\{f: \int_{0}^{\infty} f^{2}(x) d x<\right.$ $\infty\}$, i.e., the space of square integrable functions defined on $[0, \infty)$, see e.g. Szegö [19]. The inner product defined on $L_{2}(0, \infty)$ is $\langle f, g\rangle=\int_{0}^{\infty} f(x) g(x) d x$. Thus, one has that

$$
\left\langle\ell_{m}, \ell_{n}\right\rangle=\int_{0}^{\infty} \ell_{m}(x) \ell_{n}(x) d x= \begin{cases}1 & \text { for } m=n \\ 0 & \text { else }\end{cases}
$$

For any function $f \in L_{2}(0, \infty)$, the Fourier-Laguerre expansion of $f$ is given by

$$
f(x)=\sum_{0}^{\infty} f_{n}^{\dagger} \ell_{n}(x) ; \quad \text { where } f_{n}^{\dagger}=\left\langle f, \ell_{n}\right\rangle=\int_{0}^{\infty} f(x) \ell_{n}(x) d x
$$

The second part of (A.4) follows from the orthonormality of $\left\{\ell_{n}(x)\right\}$. Using this relation, a function $f \in L_{2}(0, \infty)$ is mapped onto a series $\left\{f_{n}^{\dagger}\right\}$, which is called a sequence of the Laguerre dagger coefficients. One can easily see that, if the Laguerre dagger coefficients are known, the values of $f(x)$ can be calculated easily via (A.4) based on the following recursion formula

$$
\ell_{n+1}(x)=\frac{1}{n+1}\left\{(2 n+1-x) \ell_{n}(x)-n \ell_{n-1}(x)\right\}, \quad n=2,3, \cdots
$$

starting with $\ell_{0}(x)=e^{-x / 2}$ and $\ell_{1}(x)=(1-x) e^{-x / 2}$.

Let the Laplace transform of $f(x)$ be $\varphi(w)=\int_{0}^{\infty} e^{-w x} f(x) d x$, and define $T_{f}^{\dagger}(u)=$ $\sum_{n=0}^{\infty} f_{n}^{\dagger} u^{n}$. It then follows that

$$
T_{f}^{\dagger}(u)=\frac{1}{1-u} \varphi\left(\frac{1}{2} \frac{1+u}{1-u}\right)
$$

Given the Laguerre dagger coefficients $\left\{f_{n}^{\dagger}\right\}$ of function $f$, we define the Laguerre sharp coefficients $\left\{f_{n}^{\#}\right\}$ as follows

$$
f_{0}^{\#}=f_{0}^{\dagger}, \quad f_{n}^{\#}=f_{n}^{\dagger}-f_{n-1}^{\dagger} \quad(n=1,2, \cdots) ; \quad f_{n}^{\dagger}=\sum_{m=0}^{n} f_{m}^{\#} .
$$

From Equation (A.6), one has

$$
T_{f}^{\#}(u)=\sum_{n=0}^{\infty} f_{n}^{\#} u^{n}=\varphi\left(\frac{1}{2} \frac{1+u}{1-u}\right) .
$$


It is shown by Keilson and Nunn [7] that using Equation (A.8), the continuum convolution of two functions can be calculated through discrete convolution of their Laguerre sharp coefficients. More specifically, for $(f * g)(x)=\int_{0}^{x} f(x-y) g(y) d y$, one has $\varphi_{f * g}(w)=\varphi_{f}(w) \varphi_{g}(w)$, where $\varphi_{f * g}(w), \varphi_{f}(w)$ and $\varphi_{g}(w)$ denote the Laplace transforms of $(f * g)(x), f(x)$ and $g(x)$ respectively, so that

$$
T_{f * g}^{\#}(u)=T_{f}^{\#}(u) T_{g}^{\#}(u)
$$

or equivalently

$$
(f * g)_{n}^{\#}=\sum_{m=0}^{n} f_{n-m}^{\#} g_{m}^{\#}
$$

Use Equation (A.9), one can easily calculate the Laguerre sharp coefficient $\left\{(f * g)_{n}^{\#}\right\}$ if $\left\{f_{n}^{\#}\right\}$ and $\left\{g_{n}^{\#}\right\}$ are known, and then inverting via Equation (A.7) and the series expansion in (A.4). Similarly, many other continuum operations are also mapped into lattice operations, including differentiation and integration. In what follows, some of these operational properties used in this paper are summarized.

Theorem A.1 (Moment Formula). Let $f(x)$ be a non-negative p.d.f. with Laguerre sharp coefficients $\left\{f_{n}^{\#}\right\}$. Let $M(i)=\int_{0}^{\infty} x^{i} f(x) d x$, one has

$$
M(i)=4^{i} \sum_{n=0}^{\infty}(-1)^{n} n^{i} f_{n}^{\#}, \quad 0 \leq i \leq 2
$$

Theorem A.2 (Convolution and Integration). Consider $r, f, g \in L_{2}(0, \infty)$ with Laguerre sharp coefficients $\left\{r_{n}^{\#}\right\},\left\{f_{n}^{\#}\right\}$ and $\left\{g_{n}^{\#}\right\}$ respectively. Then,

1. $r(x)=\int_{0}^{x} f(x-y) g(y) d y \Leftrightarrow r_{n}^{\#}=\sum_{m=0}^{n} f_{n-m}^{\#} g_{m}^{\#}$;

2. $r(x)=\int_{x}^{\infty} f(x) d x \Leftrightarrow r_{0}^{\#}-2-2 f_{0}^{\#}+4 \sum_{m=0}^{\infty}(-1)^{m} f_{m}^{\#}$

$$
r_{n}^{\#}-2 f_{n}^{\#}+4 \sum_{m=0}^{\infty}(-1)^{m} f_{m+n}^{\#}, n \geq 1 \text {. }
$$

\section{Matrix Laguerre transfrom}

The Laguerre transform is extended to matrix functions by Sumita [13,14]. For a $K \times K$ matrix function $\underline{\underline{a}}(x)=\left[a_{i j}(x)\right]$, let $\underline{\underline{L}}_{2}(0, \infty)=\left\{\underline{\underline{a}}(x): a_{i j}(x) \in L_{2}(0, \infty)\right\}$. The space $\underline{\underline{L}}_{2}(0, \infty)$ has inner product

$$
\langle\underline{\underline{a}}(x), \underline{\underline{b}}(x)\rangle=\frac{1}{K} \int_{0}^{\infty} \operatorname{tr}\left\{\underline{\underline{a}}(x) \underline{\underline{b}}(x)^{\top}\right\} d x .
$$

The set of matrix function $\underline{\underline{\ell}}_{n}(x)$ with $\underline{\underline{\ell}}_{n}(x)=\ell_{n}(x) \underline{\underline{I}}$ becomes an orthonormal basis of $\underline{\underline{L}}_{2}(0, \infty)$ in the following sence. There exists a unique sequence of the Laguerre dagger coefficient matrices $\left\{\underline{\underline{a}}_{n}^{\dagger}\right\}$ such that

$$
\underline{\underline{a}}(x)=\sum_{n=0}^{\infty} \underline{\underline{a}}_{n}^{\dagger} \underline{\underline{\ell}}_{n}(x) ; \quad \underline{\underline{a}}_{n}^{\dagger}=\left\langle\underline{\underline{a}}(x), \underline{\underline{\ell}}_{n}(x)\right\rangle=\int_{0}^{\infty} \underline{\underline{a}}(x) \underline{\underline{\ell}}_{n}(x) d x .
$$

Similar to the one dimensional case, the matrix Laplace transform $\underline{\underline{\alpha}}(w)=\int_{0}^{\infty} e^{-w x} \underline{\underline{a}}(x) d x$ has the relations that

$$
\underline{\underline{T}}_{a}^{\dagger}(u)=\sum_{n=0}^{\infty} \underline{\underline{a}}_{n}^{\dagger} u^{n}=\frac{1}{1-u} \underline{\underline{\alpha}}\left(\frac{1}{2} \frac{1+u}{1-u}\right)
$$


and

$$
\underline{\underline{T}}_{a}^{\#}(u)=\sum_{n=0}^{\infty} \underline{\underline{a}}_{n}^{\#} u^{n}=\underline{\underline{\alpha}}\left(\frac{1}{2} \frac{1+u}{1-u}\right),
$$

where $\underline{\underline{a}}_{n}^{\#}=\underline{\underline{a}}_{n}^{\dagger}-\underline{\underline{a}}_{n-1}^{\dagger}$.

\section{Bivariate Laguerre transfrom}

The bivariate version of the Laguerre transform is introduced in Sumita and Kijima [16]. Let $L_{2}\left(\mathbb{R}_{+}^{2}\right)=\left\{f: \iint_{\mathbb{R}_{+}^{2}} f^{2}(x, y) d x d y<\infty\right\}$ and define

$$
\ell_{m n}(x, y)=\ell_{m}(x) \ell_{n}(y), \quad 0 \leq x, y<\infty .
$$

It is then easy to see that $\left\{\ell_{m n}(x, y)\right\}$ is an orthonormal basis of $L_{2}\left(\mathbb{R}_{+}^{2}\right)$. For any $f \in$ $L_{2}\left(\mathbb{R}_{+}^{2}\right)$, the Laguerre dagger coefficients $\left\{f_{m n}^{\dagger}\right\}$ and the Laguerre sharp coefficients $\left\{f_{m n}^{\#}\right\}$ are given by

$$
\begin{aligned}
& f_{m n}^{\dagger}=\left\langle f, \ell_{m n}\right\rangle=\iint_{\mathbb{R}_{+}^{2}} f(x, y) \ell_{m n}(x, y) d x d y, \\
& f_{m n}^{\#}=f_{m n}^{\dagger}-f_{m, n-1}^{\dagger}-f_{m-1, n}^{\dagger}+f_{m-1, n-1}^{\dagger} .
\end{aligned}
$$

One then has

$$
f(x, y)=\sum_{n=0}^{\infty} \sum_{m=0}^{\infty} f_{m n}^{\dagger} \ell_{m n}(x, y) .
$$

Define the double Laplace transform $\varphi(w, s)=\iint_{\mathbb{R}_{+}^{2}} e^{-w x} e^{-s y} f(x, y) d x d y$, it follows that

$$
\begin{aligned}
& T^{\dagger}(u, v)=\sum_{n=0}^{\infty} \sum_{m=0}^{\infty} f_{m n}^{\dagger} u^{m} v^{n}=\frac{1}{1-u} \frac{1}{1-v} \varphi\left(\frac{1}{2} \frac{1+u}{1-u}, \frac{1}{2} \frac{1+v}{1-v}\right), \\
& T^{\#}(u, v)=\sum_{n=0}^{\infty} \sum_{m=0}^{\infty} f_{m n}^{\#} u^{m} v^{n}=\varphi\left(\frac{1}{2} \frac{1+u}{1-u}, \frac{1}{2} \frac{1+v}{1-v}\right) .
\end{aligned}
$$

Theorem A.3 (Bivariate Matrix Convolution and Integration). Let $\underline{a}(x, y), \underline{b}(x, y), \underline{c}(x, y) \in$ $\underline{\underline{L}}_{2}\left(\mathbb{R}_{+}^{2}\right)$ has matrix Laguerre coefficients $\left\{\underline{\underline{a}}_{m n}^{\dagger}\right\},\left\{\underline{\underline{a}}_{m n}^{\#}\right\},\left\{\underline{\underline{b}}_{m n}^{\dagger}\right\},\left\{\underline{\underline{b}}_{m n}^{\#}\right\},\left\{\underline{\underline{c}}_{m n}^{\dagger}\right\}$ and $\left\{\underline{\underline{c}}_{m n}^{\#}\right\}$ respectively. Then,

1. $\underline{\underline{a}}(x, y)=\int_{0}^{x} \int_{0}^{y} \underline{\underline{b}}\left(x-x^{\prime}, y-y^{\prime}\right) \underline{\underline{c}}\left(x^{\prime}, y^{\prime}\right) d x^{\prime} d y^{\prime}$

$$
\Leftrightarrow \quad \underline{a}_{m n}^{\#}=\sum_{i=0}^{m} \sum_{j=0}^{n} \underline{\underline{b}}_{m-i, n-j}^{\#} \underline{\underline{c}}_{i, j}^{\#} ;
$$

2. $\underline{\underline{a}}(x, y)=\int_{x}^{\infty} \underline{\underline{b}}\left(x^{\prime}, y\right) d x^{\prime} \Leftrightarrow \underline{\underline{a}}_{m n}^{\dagger}=2 \sum_{i=m+1}^{\infty} \sum_{j=0}^{n}(-1)^{m+i} \underline{\underline{b}}_{i j}^{\#}$;

3. $\underline{\underline{a}}(x, y)=\int_{y}^{\infty} \underline{\underline{b}}\left(x, y^{\prime}\right) d y^{\prime} \Leftrightarrow \quad \underline{a}_{m n}^{\dagger}=2 \sum_{i=0}^{m} \sum_{j=n+1}^{\infty}(-1)^{n+j} \underline{\underline{b}}_{i j}^{\#}$.

At the last of this section, we summarize the Laguerre coefficients of exponential density in a simple closed form, which are often appeared in applied probability theory.

\section{Exponential density}

The p.d.f. of an exponential density function having mean $\mu^{-1}$ is denoted by

$$
e(\mu ; x)=\mu e^{-\mu x}, \quad x \geq 0, \mu>0,
$$

and the corresponding Laguerre dagger coefficients $\left\{e_{n}^{\dagger}(\mu)\right\}$ is given as

$$
e_{n}^{\dagger}(\mu)=\frac{2 \mu}{2 \mu+1}\left(\frac{2 \mu-1}{2 \mu+1}\right)^{n}, \quad n \geq 0 \text {. }
$$




\section{B. Algorithms in Section 4}

In this section, provided are the numerical algorithms for evaluating the probability mass function $z_{0}(t)$ and the p.d.f. $f_{Z_{+}}(z, t)$ discussed in Section 3 , as well as the equilibrium unit premium $c_{\left[K_{a}, K_{d}\right]}^{*}$ represented by Equation (2.8) in Section 2.

For numerical evaluation, the infinite series' involved in (3.12) through (3.15) have to be truncated. Let $K$ and $L$ be the truncation points determined as

$$
\begin{aligned}
& \widetilde{\zeta}_{0}(s)=\underline{p}^{\top}(0) \times\left\{\underline{\underline{I}}+\sum_{\ell=1}^{L}\left[\alpha_{i j}\left(s+\lambda_{i}\right)\right]_{i, j \in \mathcal{J}}^{\ell}\right\} \times\left[\frac{1-\alpha_{i}\left(s+\lambda_{i}\right)}{s+\lambda_{i}}\right]_{i \in \mathcal{J}}, \\
& \widetilde{\zeta}_{+}(r, s)=\underline{p}^{\top}(0) \times\left\{\underline{\underline{I}}+\sum_{\ell=1}^{L} \underline{\widehat{\beta}}_{+}\left(s \underline{1}, \underline{\theta}_{M}(r)\right)^{\ell}\right\} \times \underline{\widehat{\beta}}_{+: D}^{*}\left(s \underline{1}, \underline{\theta}_{M}(r)\right) \times \underline{1}
\end{aligned}
$$

with

$$
\widetilde{\widehat{\beta}}_{+: i j}\left(s, \theta_{M: i}(r)\right)=\sum_{k=1}^{K} \theta_{M: i}^{k}(r) \int_{0}^{\infty} e^{-s t} a_{i j}(t) e^{-\lambda_{i} t} \frac{\left(\lambda_{i} t\right)^{k}}{k !} d t
$$

and

$$
\widetilde{\widehat{\beta}}_{+: i}^{*}\left(s, \theta_{M: i}(r)\right)=\sum_{k=1}^{K} \theta_{M: i}^{k}(r) \int_{0}^{\infty} e^{-s t} \bar{A}_{i}(t) e^{-\lambda_{i} t} \frac{\left(\lambda_{i} t\right)^{k}}{k !} d t
$$

$K$ and $L$ are determined so as to achieve the accuracy of $\epsilon$ for the first two moments of the size of each default, which is exponentially distributed with parameter $\mu$ based on (A.10).

We are now in a position to describe the main algorithm. The following operators are employed.

\section{Definition B.1.}

MMT1 : $\operatorname{MMT1}\left(\left\{f_{n}\right\}_{n: N}\right)=4 \sum_{n=0}^{N}(-1)^{n} n f_{n}$

MMT2 : $\operatorname{MMT2}\left(\left\{f_{n}\right\}_{n: N}\right)=4^{2} \sum_{n=0}^{N}(-1)^{n} n^{2} f_{n}$

$$
\begin{aligned}
\mathrm{CONV}: & \left\{c_{n}\right\}_{n: N}=\operatorname{CONV}\left(\left\{a_{n}\right\}_{n: N},\left\{b_{n}\right\}_{n: N}\right) \Leftrightarrow c_{n}=\sum_{m=0}^{n} a_{n-m} b_{m} \\
& \left.\operatorname{CONV}_{i=1}^{K}\left(\left\{f_{i: n}\right\}_{n: N}\right)=\operatorname{CONV}\left(\operatorname{CONV}_{i=1}^{K-1}\left(\left\{f_{i: n}\right\}_{n: N}\right),\left\{f_{K: n}\right\}_{n: N}\right)\right)
\end{aligned}
$$

PSUM : $\quad\left\{b_{n}\right\}_{n: N}=\operatorname{PSUM}\left(\left\{a_{n}\right\}_{n: N}\right) \Leftrightarrow b_{n}=\sum_{m=0}^{n} a_{m}$ 
OUTP : $\quad\left\{c_{(m, n)}\right\}_{m n: M N}=\operatorname{OUTP}\left(\left\{a_{m}\right\}_{m: M},\left\{b_{n}\right\}_{n: N}\right) \Leftrightarrow c_{(m, n)}=a_{m} b_{n}$

$\operatorname{BDIFF}: \quad\left\{b_{(m, n)}\right\}_{m n: M N}=\operatorname{BDIFF}\left(\left\{a_{(m, n)}\right\}_{m n: M N}\right)$

$$
\Leftrightarrow \quad b_{(m, n)}=a_{(m, n)}-a_{(m-1, n)}-a_{(m, n-1)}+a_{(m-1, n-1)}
$$

$\operatorname{VCONV}: \quad\left\{c_{n}\right\}_{n: N}=\operatorname{VCONV}\left(\left\{\underline{a}_{n}\right\}_{n: N},\left\{\underline{b}_{n}\right\}_{n: N}\right) \Leftrightarrow c_{n}=\sum_{m=0}^{n} \underline{a}_{n-m}^{\top} \underline{b}_{m}$

$\operatorname{VBCONV}: \quad\left\{c_{(m, n)}\right\}_{m n: M N}=\operatorname{VBCONV}\left(\left\{\underline{a}_{(m, n)}\right\}_{m n: M N},\left\{\underline{b}_{(m, n)}\right\}_{m n: M N}\right)$

$$
\Leftrightarrow \quad c_{(m, n)}=\sum_{i=0}^{m} \sum_{j=0}^{n} \underline{a}_{(m-i, n-j)}^{\top} \underline{b}_{(i, j)}
$$

$\operatorname{VMCONV}: \quad\left\{\underline{c}_{n}\right\}_{n: N}=\operatorname{VMCONV}\left(\left\{\underline{a}_{n}\right\}_{n: N},\left\{\underline{b}_{n}\right\}_{n: N}\right) \Leftrightarrow \quad \underline{c}_{n}^{\top}=\sum_{m=0}^{n} \underline{a}_{n-m}^{\top} \underline{\underline{b}}_{m}$

$\operatorname{VMBCONV}: \quad\left\{\underline{c}_{(m, n)}\right\}_{m n: M N}=\operatorname{VMBCONV}\left(\left\{\underline{a}_{(m, n)}\right\}_{m n: M N},\left\{\underline{\underline{b}}_{(m, n)}\right\}_{m n: M N}\right)$

$$
\Leftrightarrow \quad \underline{c}_{(m, n)}^{\top}=\sum_{i=0}^{m} \sum_{j=0}^{n} \underline{a}_{(m-i, n-j)}^{\top} \underline{b}_{(i, j)}
$$

$\operatorname{BPSUM}: \quad\left\{b_{(m, n)}\right\}_{m n: M N}=\operatorname{BPSUM}\left(\left\{a_{(m, n)}\right\}_{m n: M N}\right) \Leftrightarrow b_{(m, n)}=\sum_{i=0}^{m} \sum_{j=0}^{n} a_{(i, j)}$

$\operatorname{BINNP}: \operatorname{BINNP}\left(\left\{a_{(m, n)}\right\}_{m n: M N},\left\{b_{(m, n)}\right\}_{m n: M N}\right)=\sum_{m=0}^{M} \sum_{n=0}^{N} a_{(m, n)} b_{(m, n)}$

$\operatorname{MSUV}: \quad\left\{b_{(m, n)}\right\}_{m n: M N}=\operatorname{MSUV}\left(\left\{a_{(m, n)}\right\}_{m n: M N}\right)$

$$
\Leftrightarrow \quad b_{(m, n)}=2 \sum_{i=m+1}^{M} \sum_{j=0}^{n}(-1)^{m+i} a_{(i, j)}
$$

In what follows, the algorithm of evaluating $z_{0}(t)$ and $f_{Z_{+}}(z, t)$ is provided.

Algorithm B.2 Evaluating $z_{0}(t)$ and $f_{Z_{+}}(z, t)$ for given $K$ and $L$

Input: $p(0),\left\{y_{i: m}^{\dagger}\right\}_{m: M},\left\{y_{i: m}^{\#}\right\}_{m: M},\left\{a_{k: i j: n}^{\dagger}\right\}_{n: N},\left\{a_{k: i j: n}^{\#}\right\}_{n: N},\left\{\bar{A}_{k: i: n}^{\dagger}\right\}_{n: N},\left\{\bar{A}_{k: i: n}^{\#}\right\}_{n: N}(i, j \in$ $\mathcal{J}, 1 \leq \bar{k} \leq K), \varepsilon_{M}, \varepsilon_{N}, z, t$

Output: $z_{0}(t)$ and $f_{Z_{+}}(z, t)$

Procedure:

STEP 1: Preparation 
1: Fix $K$ and $L$

2: Initialize $M$ and $N$

\section{STEP 2: Determine $M$}

1: Evaluate the following two variable from mathematical moments of $Y_{M: i: \bullet}$ $E_{i: M}^{1}=K(L+1) \mathrm{E}\left[Y_{M: i: \bullet}\right]$ $E_{i: M}^{2}=K(L+1) \operatorname{Var}\left[Y_{M: i: \bullet}\right]+K^{2}(L+1)^{2} \mathrm{E}\left[Y_{M: i: \bullet}\right]^{2}$

2: Evaluate the corresponding approximations from the Laguerre coefficients $\left\{y_{i: m}^{\#: K L}\right\}_{m: M}=\operatorname{CONV}_{k=1}^{K(L+1)}\left(\left\{y_{i: m}^{\#}\right\}_{m: M}\right)$ $\widetilde{E}_{i: M}^{1}=\operatorname{MMT} 1\left(\left\{y_{i: m}^{\#: K L}\right\}_{m: M}\right), \widetilde{E}_{i: M}^{2}=\operatorname{MMT} 2\left(\left\{y_{i: m}^{\#: K L}\right\}_{m: M}\right)$

3: while $\max _{i \in \mathcal{J}}\left(\widetilde{E}_{i: M}^{1}-E_{i: M}^{1}, \widetilde{E}_{i: M}^{2}-E_{i: M}^{2}\right)>\varepsilon_{M}$ do

4: $\quad M=M+1$

5: Reevaluate $\left\{y_{i: m}^{\#}\right\}_{m: M}$

6: Update $\left\{y_{i: m}^{\#: K L}\right\}_{m: M}$ and reevaluate $\widetilde{E}_{i: M}^{1}, \widetilde{E}_{i: M}^{2}$

7: end while

\section{STEP 3: Determine $N$}

1: Evaluate the following two variable

$$
\begin{aligned}
& E_{i: N}^{1}=(L+1) \int_{0}^{\infty} t a_{i}(t) e^{-\lambda_{i} t} \frac{\left(\lambda_{i} t\right)^{K}}{K !} d t \\
& E_{i: N}^{2}=(L+1) \int_{0}^{\infty} t^{2} a_{i}(t) e^{-\lambda_{i} t} \frac{\left(\lambda_{i} t\right)^{K}}{K !} d t
\end{aligned}
$$

2: Evaluate the corresponding approximations from the Laguerre coefficients

$$
\begin{aligned}
& \left\{a_{K: i j: n}^{\#: K L}\right\}_{n: N}=\operatorname{CONV}_{\ell=1}^{(L+1)}\left(\left\{a_{K: i j: n}^{\#}\right\}_{n: N}\right) \\
& \widetilde{E}_{i: N}^{1}=\operatorname{MMT} 1\left(\left\{a_{K: i j: n}^{\#: K L}\right\}_{n: N}\right), \quad \widetilde{E}_{i: N}^{2}=\operatorname{MMT} 2\left(\left\{a_{K: i j: n}^{\#: K L}\right\}_{n: N}\right)
\end{aligned}
$$

3: while $\max _{i \in \mathcal{J}}\left(\widetilde{E}_{i: N}^{1}-E_{i: N}^{1}, \widetilde{E}_{i: N}^{2}-E_{i: N}^{2}\right)>\varepsilon_{N}$ do

4: $\quad N=N+1$

5: $\quad$ Reevaluate $\left\{a_{K: i j: n}^{\#}\right\}_{n: N}$

6: Update $\left\{a_{K: i j: n}^{\#: K L}\right\}_{n: N}$ and reevaluate $\widetilde{E}_{i: N}^{1}, \widetilde{E}_{i: N}^{2}$

7: end while

STEP 4: Evaluate $\left\{\underline{\underline{b}}_{+:(m, n)}^{\#}\right\}_{m n: M N}$ and $\left\{\underline{b}_{+:(m, n)}^{* \#}\right\}_{m n: M N}$

1: Evaluate $\left\{b_{+: i j:(m, n)}^{\#}\right\}_{m n: M N}$ and $\left\{b_{+: i:(m, n)}^{* \#}\right\}_{m n: M N}$ for $i \in \mathcal{J}$

$$
\begin{aligned}
& \left\{b_{+: i j:(m, n)}^{\dagger}\right\}_{m n: M N}=\sum_{k=0}^{K} \operatorname{OUTP}\left(\operatorname{PSUM}\left(\operatorname{CONV}_{q=1}^{k}\left(\left\{y_{i: m}^{\#}\right\}_{m: M}\right)\right), \operatorname{PSUM}\left(\left\{a_{k: i j: n}^{\#}\right\}_{n: N}\right)\right) \\
& \left\{b_{+: i j:(m, n)}^{\#}\right\}_{m n: M N}=\operatorname{BDIFF}\left(\left\{b_{i j:(m, n)}^{\dagger}\right\}_{m n: M N}\right) \\
& \left\{b_{+: i:(m, n)}^{* \dagger}\right\}_{m n: M N}=\sum_{k=0}^{K} \operatorname{OUTP}\left(\operatorname{PSUM}\left(\operatorname{CONV}_{q=1}^{k}\left(\left\{y_{i: m}^{\#}\right\}_{m: M}\right)\right), \operatorname{PSUM}\left(\left\{\bar{A}_{k: i: n}^{* \#}\right\}_{n: N}\right)\right) \\
& \left\{b_{i:(m, n)}^{* \#}\right\}_{m n: M N}=\operatorname{BDIFF}\left(\left\{b_{i:(m, n)}^{* \dagger}\right\}_{m n: M N}\right)
\end{aligned}
$$

2: Construct $\left\{\underline{\underline{b}}_{+:(m, n)}^{\#}\right\}_{m n: M N}$ and $\left\{\underline{b}_{+:(m, n)}^{* \#}\right\}_{m n: M N}$

STEP 5: Evaluate $z_{0}(t)$ and $f_{Z_{+}}(z, t)$ 
1: Evaluate $\left\{\underline{\pi}_{0: n}^{\#}\right\}_{n: N},\left\{\Pi_{0: n}^{\#}\right\}_{n: N},\left\{\underline{\pi}_{+:(m, n)}^{\#}\right\}_{m n: M N}$ and $\left\{\Pi_{+:(m, n)}^{\#}\right\}_{m n: M N}$ $\underline{\pi}_{0: n}^{\# \top}(1)=\underline{p}^{\top}(0) \cdot \underline{\underline{a}}_{0: n}^{\#}, \quad n=0,1, \cdots, N$ $\left\{\underline{\pi}_{0: n}^{\#}(\ell)\right\}_{n: N}=\operatorname{VMCONV}\left(\left\{\underline{\pi}_{0: n}^{\#}(\ell-1)\right\}_{n: N},\left\{\underline{\underline{a}}_{0: n}^{\#}\right\}_{n: N}\right)$

$\left\{\underline{\pi}_{0: n}^{\# \top}\right\}_{n: N}=\sum_{\ell=1}^{L}\left\{\underline{\pi}_{0: n}^{\#}(\ell)\right\}_{n: N}$

$\Pi_{0: n}^{\#}=\underline{p}^{\top}(0) \cdot \underline{\bar{A}}_{0: n}^{\#}, \quad n=0,1, \cdots, N$

$\underline{\pi}_{+:(m, n)}^{\# \top}(1)=\underline{p}^{\top}(0) \cdot \underline{\underline{b}}_{+:(m, n)}^{\#}, \quad m=0,1, \cdots, M, n=0,1, \cdots, N$

$\left\{\underline{\pi}_{+:(m, n)}^{\#}(\ell)\right\}_{m n: M N}=\operatorname{VMBCONV}\left(\left\{\underline{\pi}_{+:(m, n)}^{\#}(\ell-1)\right\}_{m n: M N},\left\{\underline{\underline{b}}_{+:(m, n)}^{\#}\right\}_{m n: M N}\right)$

$\left\{\underline{\pi}_{+:(m, n)}^{\#}\right\}_{m n: M N}=\sum_{\ell=1}^{L}\left\{\underline{\pi}_{+:(m, n)}^{\#}(\ell)\right\}_{m n: M N}$

$\Pi_{+:(m, n)}^{\#}=\underline{p}^{\top}(0) \cdot \underline{b}_{+:(m, n)}^{* \#}, \quad m=0,1, \cdots, M, n=0,1, \cdots, N$

2: Evaluate $\left\{z_{0: n}^{\#}\right\}_{n: N}$

$\left\{z_{0: n}^{\#}\right\}_{n: N}=\left\{\Pi_{0: n}^{\#}\right\}_{n: N}+\operatorname{VCONV}\left(\left\{\underline{\pi}_{0: n}^{\#}\right\}_{n: N},\left\{\underline{A}_{0: n}^{\#}\right\}_{n: N}\right)$

3: Evaluate $\left\{z_{+:(m, n)}^{\#}\right\}_{m n: M N}$

$\left\{z_{+:(m, n)}^{\#}\right\}_{m n: M N}=\left\{\Pi_{+:(m, n)}^{\#}\right\}_{m n: M N}+\operatorname{VBCONV}\left(\left\{\underline{\pi}_{+:(m, n)}^{\#}\right\}_{m n: M N},\left\{\underline{b}_{+:(m, n)}^{* \#}\right\}_{m n: M N}\right)$

4: Evaluate $z_{0}(t)$

$z_{0}(t)=\operatorname{INNP}\left(\operatorname{PSUM}\left(\left\{z_{0: n}^{\#}\right\}_{n: N}\right),\left\{\ell_{n}(t)\right\}_{n: N}\right)$

5: Evaluate $f_{Z_{+}}(z, t)$

$f_{Z_{+}}(z, t)=\operatorname{BINNP}\left(\operatorname{BPSUM}\left(\left\{z_{+:(m, n)}^{\#}\right\}_{m n: M N}\right),\left\{\ell_{(m, n)}(z, t)\right\}_{m n: M N}\right)$

where $\ell_{(m, n)}(z, t)=\ell_{m}(z) \ell_{n}(t)$

This algorithm enables one to evaluate the survival function of $Z(t)$. Let

$$
\bar{F}_{Z}(z, t)=\left\{\begin{array}{ll}
\mathrm{P}[Z(t)>z] & \text { for } z>0 \\
\mathrm{P}[Z(t)>0]+z_{0}(t) & \text { for } z=0
\end{array} .\right.
$$

Let $\left\{\bar{z}_{(m, n)}^{\dagger}\right\}_{m n: M N}$ be defined as

$$
\left\{\bar{z}_{(m, n)}^{\dagger}\right\}_{m n: M N}=\operatorname{MSUV}\left(\left\{z_{+:(m, n)}^{\#}\right\}_{m n: M N}\right)
$$

one then has

$$
\bar{F}_{Z}(z, t)=\left\{\begin{array}{ll}
\operatorname{BINNP}\left(\left\{\bar{z}_{(m, n)}^{\dagger}\right\}_{m n: M N},\left\{\ell_{(m, n)}(z, t)\right\}_{m n: M N}\right) & \text { for } z>0 \\
\operatorname{BINNP}\left(\left\{\bar{z}_{(m, n)}^{\dagger}\right\}_{m n: M N},\left\{\ell_{(m, n)}(0, t)\right\}_{m n: M N}\right)+z_{0}(t) & \text { for } z=0
\end{array} .\right.
$$

Similarly, the expectation $\mathrm{E}\left[L_{\left[K_{a}, K_{d}\right]}(t)\right]$ in Equation (2.9) can be obtained by

$$
\begin{aligned}
\mathrm{E}\left[L_{\left[K_{a}, K_{d}\right]}(t)\right]= & \operatorname{BINNP}\left(\operatorname{MSUV}\left(\operatorname{BDIFF}\left(\left\{\bar{z}_{(m, n)}^{\dagger}\right\}_{m n: M N}\right)\right),\left\{\ell_{(m, n)}\left(K_{a}, t\right)\right\}_{m n: M N}\right) \\
& +\operatorname{BINNP}\left(\operatorname{MSUV}\left(\operatorname{BDIFF}\left(\left\{\bar{z}_{(m, n)}^{\dagger}\right\}_{m n: M N}\right)\right),\left\{\ell_{(m, n)}\left(K_{d}, t\right)\right\}_{m n: M N}\right) \\
& +K_{a} \bar{F}_{Z}\left(K_{d}, t\right) .
\end{aligned}
$$


Then, one can evaluate the equilibrium unit premium $c_{\left[K_{a}, K_{d}\right]}^{*}$ in Equation (2.8) by using (B.7) repeatedly.

\author{
Jia-Ping Huang \\ VU University Amsterdam \\ De Boelelaan 1105 \\ 1081 HV Amsterdam \\ The Netherlands \\ E-mail: j.huang@vu.nl
}

